\title{
Mental Rotation and Orientation-Dependence in Shape Recognition
}

\author{
Michael J. Tarr and Steven Pinker
}

Department of Brain and Cognitive Sciences, Massachusetts Institute of Technology

How do we recognize objects despite differences in their retinal projections when they are seen at different orientations? Marr and Nishihara (1978) proposed that shapes are represented in memory as structural descriptions in objectcentered coordinate systems, so that an object is represented identically regardless of its orientation. An alternative hypothesis is that an object is represented in memory in a single representation corresponding to a canonical orientation, and a mental rotation operation transforms an input shape into that orientation before input and memory are compared. A third possibility is that shapes are stored in a set of representations, each corresponding to a different orientation. In four experiments, subjects studied several objects each at a single orientation, and were given extensive practice at naming them quickly, or at classifying them as normal or mirror-reversed, at several orientations. At first, response times increased with departure from the study orientation, with a slope similar to those obtained in classic mental rotation experiments. This suggests that subjects made both judgments by mentally transforming the orientation of the input shape to the one they had initially studied. With practice, subjects recognized the objects almost equally quickly at all the familiar orientations. At that point they were probed with the same objects appearing at novel orientations. Response times for these probes increased with increasing disparity from the previously trained orientations. This indicates that subjects had stored representations of the shapes at each of the practice orientations and recognized shapes at the new orientations by rotating them to one of the stored orientations. The results are consistent with a hybrid of the second (mental transformation) and third (multiple view) hypotheses of shape recognition: input shapes are transformed to a stored view, either the one at the nearest orientation or one at a canonical orientation. Interestingly, when mirrorimages of trained shapes were presented for naming, subjects took the same time at all orientations. This suggests that mental transformations of orientation can take the shortest path of rotation that will align an input shape and its memorized counterpart, in this case a rotation in depth about an axis in the picture plane. 1989 Academic Press, Inc.

The first author was supported by a NSF Graduate Fellowship and a James R. Killian Fellowship. This research was supported by NSF Grant BNS 8518774, and by a grant from the Alfred P. Sloan Foundation to the MIT Center for Cognitive Science. We thank Jigna Desai, Anthony Fodor, Bret Harsham, Joseph Loebach, and Dennis Vidach for their help in conducting the research; David Plotkin, Doug Wittington, and Kevin Ackley for their technical help; and David Irwin, Ellen Hildreth, Kyle Cave, Jacob Feldman, Stephen Palmer, Irvin Rock, Asher Koriat, Michael Corballis, Shimon Ullman, Larry Parsons, and an anonymous reviewer for their comments. Requests for reprints should be sent to Michael J. Tarr at E10-106, MIT, Cambridge, MA 02139. 
How do we recognize an object despite the differences in its retinal projections when it is seen at different orientations, sizes, and positions? Clearly we must compare what we see with what we remember in a way that neutralizes the effects of our viewing position, but this can be realized in different ways. There are different ways in which we could represent an input object before trying to recognize it, different formats for the stored memory representations used for recognition, and different kinds of processes used to find a match between the input and the stored representations.

Theories of shape recognition fall into three families (see Pinker, 1984, for a review). First, there are viewpoint-independent models, in which an object is assigned the same representation regardless of its size, orientation, or location. This class includes feature models, in which objects are represented as collections of spatially independent features such as intersections, angles, and curves, and structural-description models, in which objects are represented as hierarchical descriptions of the threedimensional spatial relationships between parts, using a coordinate system centered on the object or a part of the object. Prior to describing an input shape, a coordinate system is centered on it, based on its axis of elongation or symmetry, and the resulting "object-centered" description can be compared directly with stored shape descriptions, which use the same coordinate system (e.g., Marr \& Nishihara, 1978; Palmer, 1975). Second, there are single-view-plus-transformation models, in which an object is represented in a single orientation, usually one determined by the perspective of the viewer (a "viewer-centered" representation). In these models recognition is achieved by the use of transformation processes to convert an input representation of an object at its current orientation to a canonical orientation at which the memory representations are stored, or to transform memory representations into the orientation of the input shape. Third, there are multiple-view models in which an object is represented in a set of representations, each committed to a different familiar orientation, and an object is recognized if it matches any of them. There are also hybrid models. One combination that remedies some of the limitations of the single-view-plus-transformation and multiple-view models combines aspects of each. Objects are represented in a small number of viewpoint-specific representations, and an observed object is transformed to the size, orientation, and location of the "nearest" one.

Each kind of recognition mechanism makes specific predictions about the effect of orientation on the amount of time required for the recognition of an object. The viewpoint-independent models predict that the recognition time for a particular object will be invariant across all orientations (assuming that the time to assign a coordinate system to an input shape at different orientations is controlled). The multiple-views model makes a 
similar prediction. In contrast, the single-view-plus-transformation model, if it uses an incremental transformation process, predicts that recognition time will be monotonically dependent on the orientation difference between the observed object and the canonical stored one. A hybrid model with multiple representations plus rotation also predicts that recognition time will vary with orientation, but that recognition time will be monotonically dependent on the orientation difference between the observed object and the nearest of several stored representations. It is also possible, under the hybrid model, that one or more orientations have a "canonical" status (Palmer, Rosch, \& Chase, 1981), such as the upright oricntation, and that under some circumstances an input shape may be rotated into correspondence with the canonical view even if other stored views are nearer. If so, recognition times would exhibit two components, one dependent on the orientation difference between the observed object and the upright, the other dependent on the orientation difference between the observed object and the nearest stored orientation.

\section{PREVIOUS STUDIES OF THE RECOGNITION OF SHAPES AT DIFFERENT ORIENTATIONS}

\section{Evidence for a Mental Rotation Transformation}

Cooper and Shepard (1973) and Metzler and Shepard (1974) found several converging kinds of evidence suggesting the existence of an incremental or analog transformation process, which they called "mental rotation." First, when subjects discriminated standard from mirrorreversed shapes at a variety of orientations, they took monotonically longer for shapes that were further from the upright. Second, when subjects were given information about the orientation and identity of an upcoming stimulus and were allowed to prepare for it, the time they required was related linearly to the orientation; when the stimulus appeared, the time they took to discriminate its handedness was relatively invariant across absolute orientations. Third, when subjects were told to rotate a shape mentally and a probe stimulus was presented at a time and orientation that should have matched the instantaneous orientation of their changing image, the time they took to discriminate the handedness of the probe was relatively insensitive to its absolute orientation. Fourth, when subjects were given extensive practice at rotating shapes in a given direction and then were presented with new orientations a bit past $180^{\circ}$ in that direction, their response times were bimodally distributed, with peaks corresponding to the times expected for rotating the image the long and the short way around. These converging results show that mental rotation is a genuine transformation process, in which a shape is represented as passing through intermediate orientations before reaching the target orientation. 


\section{Evidence Interpreted as Showing that Mental Rotation Is Used to Assign Handedness but Not to Recognize Shape}

Because response times for unpredictable stimuli increase monotonically with increasing orientational disparity from the upright, people must use a mental transformation to a single orientation-specific representation to perform these tasks. However, this does not mean that mental rotation is used to recognize shapes. Cooper and Shepard's task was to distinguish objects from their mirror-image versions, not to recognize or name particular shapes. In fact, Cooper and Shepard suggest that in order for subjects to find the top of a shape before rotating it, they had to have identified it beforehand. Cooper found that the average identification times for six characters in six orientations were virtually the same at all orientations (Shepard \& Cooper, 1982, p. 120). This suggests that an orientation-free representation is used in the recognition of letters and that the mental rotation process is used only to determine handedness.

Subsequent experiments have replicated this kind of effect. Corballis, Zbrodoff, Shetzer, and Butler (1978) had subjects quickly name misoriented letters and digits; they found that the time subjects took to name normal (i.e., not mirror-reversed) versions of characters was largely independent of the orientation of the character. In a second experiment, in which subjects simply discriminated a single rotated target character from other rotated distractor characters, there was no effect of orientation under any circumstances. A related study by Corballis and Nagourney (1978) found that when subjects classified misoriented characters as letters or digits there was also only a tiny effect of orientation on decision time. White (1980) had subjects discriminate handedness, category (letter vs digit), or identity for standard or reversed versions of rotated characters. The presentation of each stimulus was preceded by a cue (sometimes inaccurate) about its handedness, category, or identity, in the three judgment tasks, respectively. In trials where the cue information was accurate, White found no effect of orientation on either category or identity judgments, either for standard or mirror-reversed characters, but did find a linear effect of orientation on handedness judgments. Simion, Bagnara, Roncato, and Umilta (1982) had subjects perform "same/different" judgments on simultaneously presented letters separated by varying amounts of rotation. In several of their experiments they found significant effects of orientation on reaction time, but the effect was too small to be attributed to mental rotation. Eley (1982) found that letter-like shapes containing a salient diagnostic feature (for example a small closed curve in one corner or an equilateral triangle in the center) were recognized equally quickly at all orientations.

On the basis of these effects, Corballis et al. (1978; see also Corballis, 
1988; Hinton \& Parsons, 1981) have concluded that under most circumstances shape recognition (up to but not including the shape's handedness) is accomplished by matching an input against a "description of a shape which is more or less independent of its angular orientation." Such a representation does not encode handedness information; it matches both standard and mirror-reversed versions of a shape equally well at any orientation. Therefore subjects must use other means to assess handedness. Hinton and Parsons suggest that handedness is inherently egocentric; observers determine the handedness of a shape by seeing which of its parts corresponds to our left and right sides when the shape is upright. Thus if a shape is misoriented, it must be mentally transformed to the upright. We call this the "Rotation-for-Handedness" hypothesis.

\section{Three Problems for the Rotation-for-Handedness Hypothesis}

At first glance the experimental data seem to relegate mental rotation to the highly circumscribed role of assigning handedness, implying that other mechanisms, presumably using object-centered descriptions or other orientation-invariant representations, are used to identify shapes. We suggest that this conclusion is premature; there are three serious problems for the Rotation-for-Handedness hypothesis.

1. Tasks allowing detection of local cues. First, in many experimental demonstrations of the orientation-invariance of shape recognition, the objects could have contained one or more diagnostic local features that allowed subjects to discriminate them without processing their shapes fully. Takano (1989) notes that shapes can possess both "orientationbound" and "orientation-free" information, and if a shape can be uniquely identified by the presence of orientation-free information, mental rotation is unnecessary. The presence of orientation-frce local diagnostic features was deliberate in the design of Eley's (1982) stimuli, and he notes that it is unclear whether detecting such features is a fundamental recognition process or a result of particular aspects of experimental tasks such as extensive familiarization with the stimuli prior to testing and small set sizes.

A similar problem may inhere in White's (1980) experiment, where the presentation of a correct information cue for either identity or category may have allowed subjects to prepare for the task by looking for a diagnostic orientation-free feature (or by activating one or more orientationspecific representations based on the cue). In contrast, the presentation of a cue for handedness would not have allowed subjects to prepare for the handedness judgment, since handedness information does not in general allow any concrete feature or shape representation to be activated beforehand. Similarly, in Corballis et al.'s second experiment, where recognition times showed no effect of orientation whatsoever, subjects sim- 
ply had to discriminate a single alphanumeric character from a set of distractors, enabling them to perform the task by looking for one or more simple features of a character (e.g., a closed semicircle for the letter “'R").

2. Persistent small effects of orientation. A second problem for the rotation-for-handedness hypothesis is the repeated finding that orientation does have a significant effect on recognition time, albeit a small one (Corballis et al., 1978; Corballis \& Nagourney, 1978; Simion et al., 1982). Corballis et al. note that the rotation rate estimated from their data is far too fast to be caused by consistent use of Cooper and Shepard's mental rotation process; they suggest that it could be due to subjects' occasional use of mental rotation to double-check the results of an orientationinvariant recognition process, resulting in a small number of orientationsensitive data being averaged with a larger number of unvarying data. However, Jolicoeur and Landau (1984) suggest that normalizing the orientation of simple shapes might be accomplished extremely rapidly, making it hard to detect strong orientation effects in chronometric data. By having subjects identify misoriented letters and digits presented for very brief durations followed by a mask, Jolicoeur and Landau were able to increase subject's identification error rates to $80 \%$ on practice letters and digits. When new characters were presented for the same duration with a mask, subjects made systematically more identification errors as characters were rotated further from upright. They estimate that as little as $15 \mathrm{~ms}$ is sufficient time to compensate for $180^{\circ}$ of rotation from the upright; this is based on their finding that an additional $15 \mathrm{~ms}$ of exposure time would eliminate errors at all orientations up to $180^{\circ}$. Jolicoeur and Landau suggest that their data support a model based on "holistic mechanisms" or "time-consuming normalization processes" other than classical mental rotation.

A defender of the rotation-for-handedness hypothesis, however, could accommodate these data. Even if representations used in recognition were completely orientation-independent, a perceiver must first find the intrinsic axes or intrinsic top of an object in order to describe it within a coordinate system centered on that object. If the search for the intrinsic axis of an input shape begins at the top of the display, rotations further from the upright would be expected to produce an increase in recognition time, and this axis-finding process could be faster than the rate of mental rotation. In fact, Carpenter and Just (1978) found in their eye-movement recordings that mental rotation consists of two phases: an orientationdependent but very rapid search for landmark parts of the to-be-rotated object, and a much slower orientation-dependent process of shape rotation itself. It is possible, then, that the extremely brief presentation durations used in Jolicoeur and Landau's (1984) experiments may have pre- 
vented subjects from locating the axes or tops of the shapes in some trials, leading to recognition errors because the object cannot be described without first locating the axes. Because of this possibility, evidence for rapid orientation-dependent processes in recognition neither confirm nor refute the rotation-for-handedness hypothesis.

3. Interaction with familiarity. A final problem for the rotationfor-handedness hypothesis is that orientation-independence in recognition time seems to occur only for highly familiar combinations of shapes and orientations; when unfamiliar stimuli must be recognized, orientation effects reminiscent of mental rotation appear. Shinar and Owen (1973) conducted several experiments in which they taught subjects a set of novel polygonal forms at an upright orientation and then had the subjects classify misoriented test shapes as being a member or not being a member of the taught set. The time to perform this old-new judgment for the familiar shapes was in fact dependent on their orientation, and this effect disappeared with practice. Jolicoeur (1985) had subjects name line drawings of natural objects. At first their naming times increased as the drawings were oriented further from the upright, with a slope comparable to those obtained in classic mental rotation tasks. With practice, the effects of orientation diminished, though the diminution did not transfer to a new set of objects. This pattern of results suggests that people indeed use mental rotation to recognize unfamiliar shapes or examples of shapes. As the objects become increasingly familiar, subjects might become less sensitive to their orientation, for one of two reasons. They could develop an orientation-invariant representation of it, such as an object-centered structural description or set of features. Takano (1989) presents this kind of explanation, suggesting that Jolicoeur's subjects may have needed practice to develop the orientation-free representations of objects that eliminate the need for mental rotation. Alternatively, subjects could come to store a set of orientation-specific representations of the object, one for each orientation it is seen at, at which point recognition of the object at any of these orientations could be done in constant time by a direct match.

These familiarity effects complicate the interpretation of all of the experiments in which subjects were shown alphanumeric characters. As Corballis et al. and others point out (Corballis \& Cullen, 1986; Jolicoeur \& Landau, 1984; Koriat \& Norman, 1985), letters and digits are highly familiar shapes that subjects have had a great deal of prior experience recognizing, presumably at many orientations. Thus it is possible that people store multiple orientation-specific representations for them; recognition times would be constant across orientations because any orientation would match some stored representation. In fact this hypothesis is consistent with most of the data from the Corballis et al. studies. In their 
experiments where subjects named standard and reversed versions of characters, although there was only a tiny effect of orientation on naming latencies for standard versions, there was a large effect of orientation on naming latencies for reversed versions. On the multiple-view hypothesis, this could be explained by the assumption that people are familiar with multiple orientations of standard characters but only a single orientation of their mirror-reversed versions, which are infrequently seen at orientations other than the upright (Corballis \& Cullen, 1986; Koriat \& Norman, 1985). In addition, it is more likely that multiple orientation-specific representations exist for standard characters within $\pm 90^{\circ}$ from upright, since subjects rarely read and write characters beyond these limits. This would explain why mental rotation functions for alphanumeric characters are generally curvilinear, with smaller effects for orientations near the upright (see Koriat and Norman, 1985). With practice, subjects should begin to develop new representations for the presented orientations of the reversed versions and for previously unstored orientations of the standard versions of characters. This would account for Corballis et al.'s (1978) finding of a decrease in the effect of orientation with practice.

How would a multiple-view model explain Cooper and Shepard's (1973) results, where mental rotation is required for handedness judgments? Why couldn't subjects simply note whether the misoriented shapes matched some stored view and respond "normal" if it did and "mirrorreversed" if it did not, independent of orientation? One possibility is that whereas each of the multiple representations does correspond to a shape in a particular handedness, which version it is (normal or mirror) is not explicitly coded in the label of the representation. Thus to make a judgment about handedness the character still must be aligned with the egocentric coordinate system in which left and right are defined. This explanation is supported by the fact that recognition times for reversed characters are consistently longer than those for standard characters (Corballis et al., 1978; Corballis \& Nagourney, 1978). This suggests that the representations used in recognition are handedness-specific, although not in a way that enables the overt determination of handedness. If so, we might expect that as subjects are given increasing practice at determining the handedness of alphanumeric characters at various orientations, they should become less sensitive to orientation, just as is found for recognition. Although Cooper and Shepard (1973) found no change in the rate of mental rotation in their handedness discrimination task even with extensive practice, their non-naive subjects may have chosen to stick with the rotation strategy at all times. Kaushall and Parsons (1981) found that when subjects performed same-different judgments on successively presented three-dimensional block structures at different orientations, slopes decreased (the rate of rotation got faster) after extensive practice (504 
trials). Furthermore, Koriat and Norman (1985) found that as subjects became familiar with a set of shapes in a handedness discrimination task, the effects of orientation for stimuli near the upright diminished. This suggests that handedness discrimination and shape recognition may not be as different as earlier studies suggested, if enough practice at performing the task with each shape at each orientation is provided.

In sum, the empirical literature does not clearly support the rotationfor-handedness hypothesis. Unless there is a local diagnostic feature serving to distinguish shapes, both handedness judgments and recognition judgments take increasingly more time for orientations farther from the upright when objects arc unfamiliar, but become ncarly (though not completely) independent of orientation as the objects become familiar. This seems to indicate a role for mental rotation in the recognition of unfamiliar stimuli; the practice/familiarity effect, however, could reflect either the gradual creation of an orientation-independent representation for each shape or the storing of a set of orientation-dependent representations, one for each shape at each orientation. Thus the question of which combination of the three classes of mechanisms people use to achieve shape recognition is unresolved.

Existing evidence, even Jolicoeur's finding that diminished effects of orientation do not transfer from a set of practiced objects to a set of new objects, cannot distinguish the possibilities. The problem is that this lack of transfer demonstrates only pattern-specificity, not orientationspecificity. Both orientation-invariant and multiple orientation-specific representations are pattern-specific, although only in the latter case are the acquired representations committed to particular orientations.

The experiments presented were designed to examine the orientationspecificity of representations of familiar and unfamiliar objects used in recognition. All of our experiments had elements that are important for testing the competing hypotheses. First, they all used novel characters that contained similar local features, but different global configurations, and therefore contained no local diagnostic features that might have provided an alternate path to recognition (for an example of this type of recognition, see Eley, 1982). Second, they all have a salient feature indicating their bottom, and a well-marked intrinsic axis, minimizing effects of finding the top-bottom axis at different orientations. Third, since subjects had no experience with these characters until participating in the experiment, it is possible to control which orientations they were familiar with. We give subjects large amounts of practice naming characters in particular orientations, at which point response times are expected to flatten out, and then we probe subjects with the same characters in new "surprise" orientations. If subjects store multiple orientation-specific representations during the practice phase, it is expected that practice 
effects will not transfer to new orientations and there will be a large effect of orientation for the surprise orientations. Alternatively, if the representations of characters stored during practice are orientation-invariant, the practice effects will transfer to new orientations and there will not be an effect of orientation on naming latencies for either practice or surprise orientations.

\section{EXPERIMENT 1}

In order to determine when mental rotation is used in shape recognition in this paper, we will be looking for orientation effects on recognition time. Although we will not attempt to replicate the many converging experiments used by Cooper and Shepard to demonstrate the use of a rotation process, we do wish to establish that the slope of the function relating response times to orientation is close to that obtained in Cooper and Shepard's experiments. This would suggest (though of course it would not prove) that a similar normalization or rotation process is being used by our subjects. To do this, however, we must first establish that our stimuli and procedures are comparable to those of Cooper and Shepard. Thus we first ran a study where subjects discriminate handedness, the task that uncontroversially involves mental rotation, to verify that our stimuli are rotated at the same rate as those used in previous experiments.

In addition, this experiment examines the effect of extensive practice on the slope of the reaction time function for handedness judgments. Although there is some evidence for such practice effects in both recognition (Corballis et al., 1978; Jolicoeur, 1985; Shinar \& Owen, 1973) and handedness judgments (Kaushall \& Parsons, 1981), no study has demonstrated practice effects for handedness judgments of two-dimensional shapes rotated in the picture plane. Finally, this experiment examines the central issue of concern: namely, whether such practice effects are pattern-specific and/or orientation-specific.

In this study, subjects make mirror-image judgments on three novel characters presented at four orientations. After a great deal of practice making handedness judgments at these four orientations, subjects were presented with four new orientations and asked to make the same judgment. It was expected that initially the effect of orientation on the latency to make a judgment would be comparable to the effect of orientation found in other mental rotation studies. We also sought to examine the effects of practice. It is possible that the representations stored with practice, although useful for recognition, do not encode handedness, as in the model proposed by Hinton and Parsons (1981). In this case we would expect to find that the effect of orientation does not diminish with practice because characters must still be aligned with an upright egocentric frame of reference to determine handedness. Alternatively, it is possible that the 
representations created with practice do have handedness information which is accessible to other processes, in which case orientation effects should decrease.

Furthermore, by surprising subjects with new orientations we are able to investigate the orientation-specificity of the stored representations or strategies responsible for any reduced effect of orientation. In particular, if orientation-specific representations that explicitly encode handedness are stored with practice, these representations would provide no benefit for performing mirror-image judgments at new, nonstored orientations. Alternatively, if an orientation-invariant representation that explicitly encodes handedness is stored with practice, any reduction in the cffect of orientation should transfer to new orientations.

\section{Method}

Subjects. Twelve students from the metropolitan Boston area participated in the experiment for pay. No subject participated more than once in any condition or experiment reported in this paper.

Materials. The stimuli consisted of seven asymmetrical characters illustrated in Fig. 1 in their upright positions. Orientations are reported in degrees measured clockwise starting from the upper vertical; hence these shapes are at $0^{\circ}$. Both the standard and the reversed versions of a character were used. Stimuli were drawn within a circle in eight orientations ( $45^{\circ}$ steps) on a monochrome CRT with a resolution of $320 \times 240$ pixels controlled by an LSI/11 microcomputer. All rotations were around the center point of the screen and the center point of the imaginary square defined by the farthest reaching points of the shape. The CRT was approximately $38 \mathrm{~cm}$ from a chin rest, and the characters were drawn in a 8.7 $\times 8.7-\mathrm{cm}$ square area on the screen, resulting in a $13.06^{\circ} \times 13.06^{\circ}$ area of visual angle. To guard against the idiosyncratic effects of particular stimuli, the characters were grouped for counterbalancing purposes into three sets of three named characters each: set $\mathrm{A}$ was composed of characters 1,2 , and 3 ; set $B$ of characters 2,3 , and 4 ; and set $C$ of characters 3,6 ,

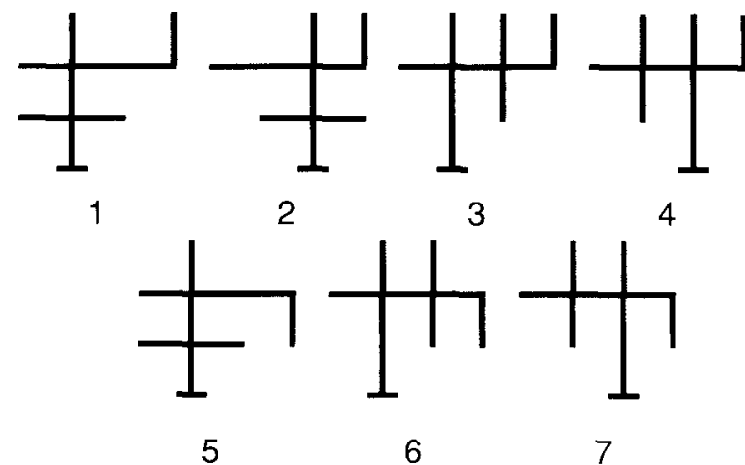

FIG. 1. Standard versions of letter-like asymmetrical characters in upright orientations. In each of these characters the main axis and bottom of the character are clearly marked by a small horizontal "foot" that is shorter than any other line segment and is the only terminating line segment. 
and 7. The first character of each set was named "Kip," the second was named "Kef," and the third was named "Kor." Each of these sets was presented to one-third of the subjects, who were not aware of the groupings.

Procedure. Subjects were shown both standard and reversed versions of the three characters that were members of the assigned set during the preliminary training session. To prevent all of the stimuli from having a common "arm" on the same side serving as a cue to handedness, subjects were given the mirror-image of the second character of each set as shown in Fig. 1 as the standard version. Subjects were shown both versions of the three characters in the assigned set on paper and traced the standard version of each of the characters five times. For each tracing the subject also was instructed to write the name of the character and repeat it aloud. Subjects were then asked to draw the standard version of each of the characters named by the experimenter. Feedback was given and subjects continued to draw the characters named until they twice correctly had drawn all three characters in sequence.

Throughout the rest of the experiment the characters were shown one at a time on the monochrome CRT. Subjects were told that they were to wait for a fixation point (a " +") and then would see one of the characters displayed in one of many orientations. They were instructed to decide as quickly as possible, while minimizing errors, whether it was a standard or reversed version of one of the characters they had learned in the training session. Subjects responded via a labeled two-key response board with the standard response corresponding to the right key and the reversed response corresponding to the left key. On trials where subjects made an error, they heard a beep.

Design. Both standard and reversed versions of the three characters were displayed in the four orientations illustrated in Fig. 2a: $0^{\circ},+45^{\circ},+135^{\circ}$, and $-90^{\circ}$. The first part of the experiment consisted of "practice" blocks of trials. Each praci "ce block contained 14 preliminary trials, randomly selected across conditions, followed by 192 trials consisting of each of the three characters in their standard and reversed versions in the four orientations each presented eight times. In the second part of the experiment trials were organized into a "surprise" block consisting of 14 random preliminary trials, followed by 384 trials composed of the same trials as the practice blocks with the addition of four new display orientations illustrated in Fig. 2a: $-45^{\circ},+90^{\circ},-135^{\circ}$, and $+180^{\circ}$. In the surprise block the 14 preliminary trials were composed of only orientations previously used in practice blocks. In all blocks the order of the trials following the 14 preliminary trials was determined randomly for each subject. Subjects were given a self-timed break every 103 trials.

Subjects were run in a total of four sessions each approximately $1 \mathrm{~h}$ long. In the first session subjects were first given the training procedure and then run in two practice blocks. Subjects were run in four practice blocks in both the second and third sessions. In the fourth session subjects were run in two practice blocks prior to the surprise block, to ensure that any effects in the surprise block were not due to a beginning-of-session effect. Not counting preliminary trials, each subject was run in a total of 96 trials for every object at a particular handedness and practice orientation.

\section{Results}

Subject's responses and reaction times were recorded by the microcomputer. Incorrect responses and responses for the 14 preliminary trials in each block were discarded and means for each orientation were calculated by block, averaging over all characters. Since clockwise and counterclockwise rotations of the same magnitude produce approximately equal reaction times in most mental rotation studies, and there is strong 

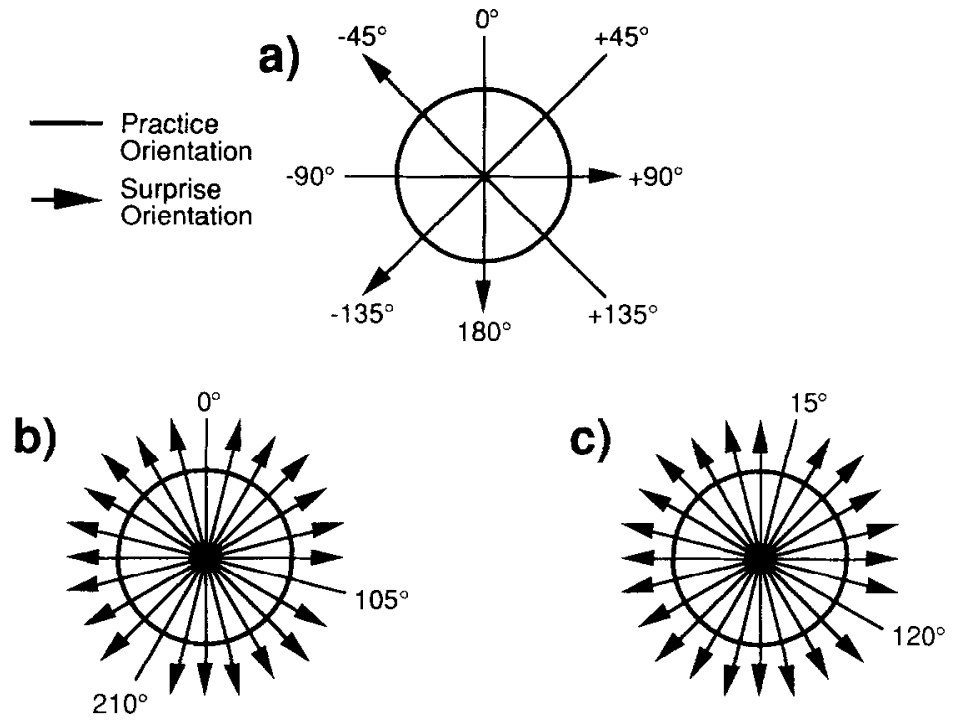

FIG. 2. (a) Angular layout of practice and surprise orientations for stimulus characters in Experiment 1 and Condition 0/45/-90/135 of Experiment 2. (b) Angular layout of practice and surprise orientations for stimulus characters in Condition 0/105/-150 of Experiment 2, Condition 0/105/-150 of Experiment 3, and Control Condition of Experiment 3. (c) Angular layout of practice and surprise orientations for stimulus characters in Condition 15/120 of Experiment 3 and Experiment 4.

evidence that subjects generally rotate the shortest way around to the upright (Cooper \& Shepard, 1973; Shepard \& Cooper, 1982), it is assumed here and throughout this paper that orientations reflected across the vertical axis are equivalent. This assumption is supported by the finding that in this and subsequent experiments mean reaction times for counterclockwise orientations fall on or near the straight line defined by the data points for clockwise orientations. Therefore the practice orientation of $-90^{\circ}$ may be treated as a $+90^{\circ}$ rotation and the surprise orientations of $-135^{\circ}$ and $-45^{\circ}$ may be treated as $+135^{\circ}$ and $+45^{\circ}$ rotations, respectively. The effect of orientation may be characterized by plotting the reaction time means against orientation and calculating the slope, measured in milliseconds per degree, of the best fitting line determined by the method of least squares.

Figure 3 shows mean reaction times for the blocks of interest, collapsed over standard and reversed versions. The slope averaged across subjects in Block 1 is $3.52 \mathrm{~ms} / \mathrm{deg}(284 \mathrm{deg} / \mathrm{s})$ of rotation. As shown in Fig. 4, over the next 11 practice blocks the combined slope steadily decreased, with the slope for Block 12 being $1.00 \mathrm{~ms} / \mathrm{deg}(1000 \mathrm{deg} / \mathrm{s})$. There was also an overall decrease in reaction time, reflecting a decrease in intercept as well 


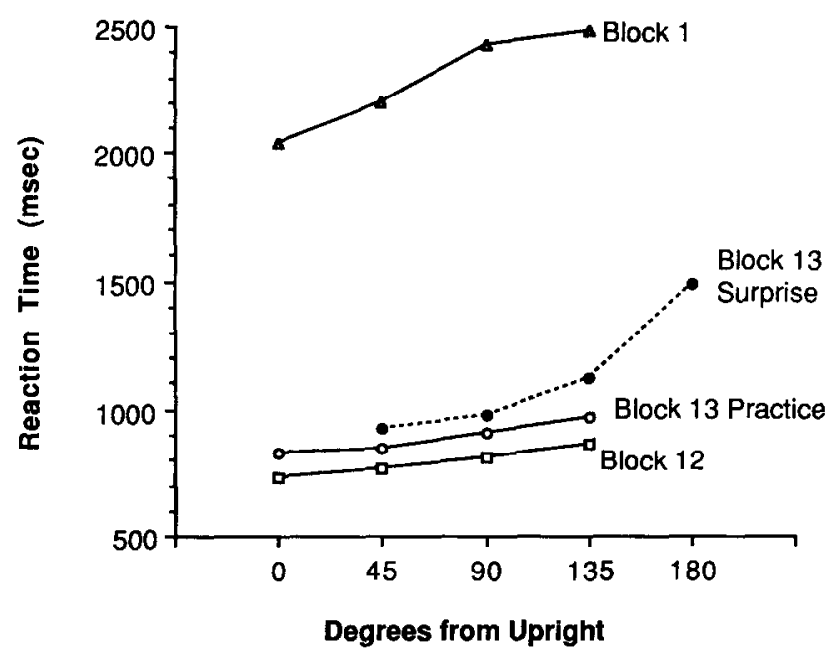

FIG. 3. Mean reaction times for performing a standard/reversed discrimination as a function of orientation collapsing over standard and reversed versions in Blocks 1, 12, and 13 of Experiment 1.

as slope, across all orientations from Block 1 to Block 12. In Block 13, the surprise block, the slope for the practice orientations was $1.04 \mathrm{~ms} / \mathrm{deg}$ $(962 \mathrm{deg} / \mathrm{s})$, while the slope for the surprise orientations was $4.08 \mathrm{~ms} / \mathrm{deg}$ $(245 \mathrm{deg} / \mathrm{s})$. Slopes broken down by standard and reversed versions did not vary significantly from this overall pattern. As Fig. 3 shows, intercepts for both surprise and practice orientations in Block 13 did not differ notably from those of the preceding block.

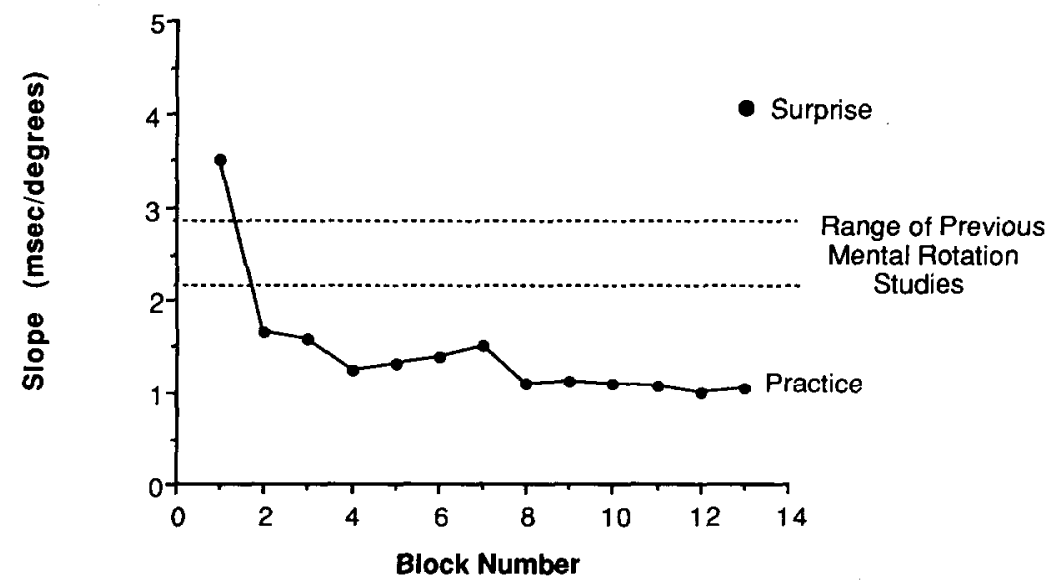

Fig. 4. Slopes for all blocks of Experiment 1 collapsed over standard and reversed versions. 
The effects of practice were investigated in a two-way analysis of variance (ANOVA) on data from the practice blocks (1-12), with Block Number and Orientation as factors. A significant main effect for Block $(F(8,88)$ $=41.30, p<.001$ ) was reflected in the data as an overall drop in reaction times with practice. A significant main effect for Orientation across blocks was found $(F(3,33)=55.83, p<.001)$, as was a significant interaction between Block Number and Orientation $(F(24,264)=4.65, p<$ .001 ), which indicated that the effect of orientation changed with practice and, as shown in the data, diminished with practice. ${ }^{1}$ A two-way ANOVA was performed to compare data from Block 1 and Block 13-Surprise, cxcluding data from $0^{\circ}$ and $180^{\circ}$ so that only rotations of cqual magnitude were compared. There was no significant interaction between the Block 1 vs Block 13-Surprise factor and Orientation, consistent with the suggestion that the diminished effects of orientation with practice did not transfer to new, surprise orientations.

A two-way ANOVA combining data from both practice and surprise orientations of equal magnitude in Block 13 (i.e., excluding $0^{\circ}$ and $180^{\circ}$ ) revealed a significant main effect of practice orientations versus surprise orientations $(F(1,11)=41.39, p<.005)$, a main effect of orientation $(F(2,22)=37.45, p<.005)$, and a significant interaction between Practice/Surprise and Orientation $(F(2,22)=5.75, p<0.01)$. Subjecting the interaction to a trend analysis over Orientation reveals a significant interaction between the Practice/Surprise factor and the linear trend of Orientation $(F(1,11)=5.39, p<0.04)$.

Error rates were 4-5\% in Block 1 and $1-6 \%$ in Block 13; error rates for different orientations never reflected a speed/accuracy tradeoff. ${ }^{2}$ Rather, here and in the other experiments reported in this paper speed and accuracy showed similar trends across orientation.

\section{Discussion}

The noteworthy results from this experiment are as follows. First, as in Cooper and Shepard (1973), subjects used an orientation-dependent transformation process to align newly learned characters with upright before making a handedness discrimination. Given the monotonicity of the reaction time function shown in Fig. 3 it is reasonable to assume that transformations took the shortest way around to the upright. Second, as in Kaushall and Parsons (1981), the effects of orientation diminished with

\footnotetext{
${ }^{1}$ Due to a loss of data for one subject for Blocks 6,8 , and 9 , these blocks were not included in these ANOVAs

${ }^{2}$ Complete error data are available from the authors upon request.
} 
practice. Third, these diminished effects of orientation did not transfer to the same characters displayed in new, never-before-seen orientations.

The rate of transformation for our stimulus characters in Block 1 is comparable with estimates from classic mental rotation studies, including those that had other converging evidence for an analog rotation process (Cooper, 1975, 1976; Cooper \& Shepard, 1973; Cooper \& Farrell, reported in Shepard \& Cooper, 1982). The range of rates obtained in these studies is shown in Fig. 4; the lower bound of the range is taken from Cooper (1975) and the upper bound is taken from Cooper and Shepard (1973), with Cooper and Farrell's results falling somewhere in between. The slope calculated from Jolicoeur's (1985) experiment on the recognition of natural objects also falls within this range. These comparisons suggest the transformation process used to align our novel characters with the upright in performing handedness judgments is the same transformation process studied in previous experiments.

In Block 1 the response time data strongly suggest that subjects use mental rotation to align characters with the upright, and in later blocks the data suggest that they no longer do so for practice orientations. Although there exists a small residual slope for these trials, this slope would translate to a hypothetical rate of rotation that is far faster than any previously found in studies that uncontroversially involve a rotation process (Shepard \& Cooper, 1982). Shinar and Owen (1973), Corballis et al. (1978), and Simion et al. (1982) obtained similar small orientation effects and ruled out mental rotation as a possible cause. These authors discuss several possible reasons why such slopes are not exactly zero. One is that on a small percentage of trials subjects still engage in a rotation strategy (e.g., Corballis et al., 1978); another is that some of the components of the response time other than mental rotation also display a slight dependency on orientation (recall that Carpenter and Just (1978) obtained eyemovement data consistent with this suggestion).

This practice effect at first appears to be inconsistent with Jolicoeur's (1985) Experiment 4, in which he found no reduction in the effect of orientation with practice in making a handedness discrimination. However, Jolicoeur's subjects received a total of only 216 trials (plus 12 preliminary trials), 36 per block. In our experiment subjects received 192 trials per block and a total of 2304 trials (excluding 168 preliminary trials). We found that decreased effects of orientation did not begin for most subjects until Block 2. In addition, Jolicoeur had his subjects make a handedness discrimination for 36 different stimuli, with each object being shown only one time at each orientation during the experiment. In contrast, we had our subjects make a handedness discrimination upon only 3 different shapes, and each object was shown 192 times at each orientation during the experiment. Thus, it appears likely that subjects in Jolicoeur's 
experiment did not have enough experience to store version-specific representations. The results of Kaushall and Parsons (1981), who also had many trials and only two shapes, are consistent with this interpretation.

Why do subjects stop mentally rotating shapes at well-learned orientations? They must have stored new handedness-specific representations for each character, contrary to the hypotheses of Corballis et al. (1978; Corballis, 1988) and Hinton and Parsons (1981) that objects are represented in an orientation-invariant format that does not encode handedness in accessible form.

The most important result of this experiment is the failure of the practice effect to transfer to new orientations. If subjects had stored handedness-specific orientation-invariant representations, the magnitude of the orientation for the surprise orientations should have had no more of an effect on judgment latency than that for well-learned orientations. But what we found for surprise orientations in Block 13 was a systematic effect of orientation on handedness judgments. This slope was comparable to the slope found in Block 1 ( 245 and $284 \mathrm{deg} / \mathrm{s}$, respectively), suggesting that for surprise orientations subjects were using mental rotation to align the observed object with the upright. Furthermore, within the same block the diminished effect of orientation on practice orientations was maintained. This suggests that in Block 13, subjects did not simply revert to a strategy used in Block 1 in response to the surprise orientations, since a shift in strategy would affect all orientations in a particular block.

Note that we were able to see evidence for mental rotation only because subjects chose to rotate to the upright orientation on some trials rather than to the nearest stored orientation; if the latter strategy had been uscd exclusively, we would have scen a flat response time function since all surprise orientations were exactly $45^{\circ}$ from a stored one. Presumably the upright orientation is canonical (Palmer et al., 1981; Rock, 1973) and attracts picture-plane rotations to it, perhaps especially when a handedness judgment must be made because it depends on an egocentric reference frame. What is not clear is why a stored representation at a nonupright orientation can be used to classify a stimulus that matches that orientation exactly, but apparently cannot be used as easily as a target for rotation of stimuli near that orientation.

These findings support the claim that with increasing experience with a shape, subjects formed representations of characters that enabled handedness judgments to be made only at specific orientations. Such representations cannot be descriptions that are so abstract that they apply equally well across rotation and mirror-reversal. Rather, they must correspond more concretely to the physical arrangement of parts in the visual field that a shape displays in a particular orientation relative to the viewer. 


\section{EXPERIMENT 2}

Experiment 1 provides evidence that with sufficient practice, subjects come to store handedness-specific, orientation-specific representations in a handedness discrimination task. As mentioned, some theories have assigned special status to information about handedness (Corballis, 1988; Corballis et al., 1978; Hinton \& Parsons, 1981). It is possible that the results obtained in Experiment 1 were task-specific and that for purposes of recognition handedness is not encoded in accessible form in the representation of the shape. That is, although we are capable of storing handedness-specific representations, we do not normally do so unless the object to be recognized must be discriminated from its reflection.

In this experiment subjects had to recognize the novel characters used in Experiment 1, none of which was a mirror-reversal of any other. Corballis et al. (1978) found that effects of orientation on naming times for alphanumeric characters are restricted primarily to mirror-reversed versions. But as mentioned, their studies used familiar stimuli which may have been encoded previously in multiple orientation-specific representations of their normal versions. We test our explanation of the orientation-independence of recognition time in the experiments of Corballis and others by examining whether the recognition of unfamiliar stimuli is orientation-dependent and whether the effect of orientation diminishes with practice.

In this experiment, subjects practiced recognizing characters long enough so that their performance was equivalent at all practice orientations. As in Experiment 1, they were then surprised with the same characters displayed in new orientations. If the practiced characters are stored as multiple orientation-specific representations, subjects should give evidence of using mental rotation to align characters in surprise orientations with stored orientation-specific representations.

One difficulty with the multiple orientation-specific representation hypothesis is that the number of stored representations for a single object may become unmanageably large to accomplish recognition at the many possible orientations from which the object may be observed. Fortunately, this is not an insurmountable problem; it may be handled by reducing the number of stored representations for an object and assuming that the stored representations are used over a range of neighboring orientations. Orientation-specific representations may be used in two ways in the recognition of unstored orientations: First, at orientations very near to a stored orientation, the changes in the observed shape of an object are minimal and may be ignored for purposes of recognition (see Koriat \& Norman, 1985). This could happen if the representation of the orientation of parts did not correspond to arbitrarily precise orientations, but spanned 
a narrow range of orientations. Second, at orientations somewhat more distant from any stored orientation, the changes in the observed shape of an object are great enough to require a transformation to align the observed object with a stored representation. Thus, mental rotation might be used not only when observing an object for the first time, but any time an object is observed in a new, previously unseen orientation sufficiently distant from any stored representations. By spacing the stored orientation-specific representations of an object at regular intervals, perhaps at a greater density for ranges of orientations at which objects are typically seen, an object may be represented for recognition by a reasonably small number of representations, yet recognized at any orientation with only minimal transformations.

Two different conditions were run. In the first condition, the practice and surprise orientations replicated those used in Experiment 1. However, in this design all the surprise orientations were the same distance away from the nearest practice orientation. As mentioned in the Discussion of Experiment 1, with this set of orientations a flat response time function would leave no way of determining whether stimuli were rotated to the nearest well-learned orientation or were not rotated at all. We eliminated this problem in the second condition, where new subjects were given practice at recognizing shapes at orientations spaced at regular intervals, and were then surprised with orientations more finely and evenly spaced throughout the range of unlearned orientations. This feature allows us to compare predictions based on rotation to the upright and rotation to familiar non-upright orientations; it also allows us to extend our findings about orientation-dependence in recognition to a wider range of conditions.

\section{Method}

Suhjects. Twenty-four students from the metropolitan Boston area participated in the experiment for pay: 12 in Condition 0/45/-90/135 and 12 in Condition 0/105/-150.

Materials. The stimulus items, computer display, stimulus sets, and experimental conditions were identical to those used in Experiment 1. In Condition 0/45/-90/135 both the practice and surprise orientations were identical to those used in Experiment 1, while in Condition $0 / 105 /-150$ the three practice orientations were $0^{\circ},+105^{\circ}$, and $-150^{\circ}\left(210^{\circ}\right)$ and the 21 surprise orientations were located in $15^{\circ}$ increments clockwise from $0^{\circ}$ (skipping the practice orientations), as illustrated in Fig. $2 b$.

Procedure. Subjects were presented with three line drawings of characters in one of the sets described in Experiment 1, and told to learn them because in the rest of the experiment they would have to recognize them in a variety of orientations on a computer screen. The training procedure was the same as in Experiment 1 except that subjects were never shown the reversed versions of the stimuli and the second character of each set was exactly as shown in Fig. 1. In addition, the four characters not used in the named set were presented during testing to the subject (the subject was not shown these distractors during the training phase), at the same orientations as the three trained characters. The four distractor char- 
acters contained the same kinds of local features as the trained characters and each other, but in different configurations. We included these distractors to minimize the possibility that subjects would find some local feature or configuration that distinguished among the three shapes in the training set, without requiring them to learn names for a large number of shapes.

Subjects responded via a three-key response board with the left key labeled "Kip," the center key labeled "Kef," and the right key labeled "Kor." Subjects were told they could use either hand or both hands to respond. They were informed that the characters would appear in many orientations and that sometimes a character they had not been taught would be displayed. In this case they were to press a footpedal.

Design. In Condition 0/45/-90/135 trials were organized into "practice" blocks of 140 trials, composed of 12 randomly selected preliminary trials, followed by 128 trials consisting of the three characters in their standard versions in four orientations (the same ones used for the practice blocks in Experiment 1), each used eight times, and the four distractor characters in the same four orientations, each used twice. The order of the trials following the 12 preliminary trials was determined randomly. Trials were organized into "surprise" blocks of 256 trials, plus 12 preliminary trials, that corresponded to the four surprise orientations and the four practice orientations used in Experiment 1. Subjects were given a self-timed break every 70 trials in each block.

In Condition $0 / 105 /-150$ trials were organized into practice blocks of 110 trials, consisting of 14 randomly selected preliminary trials, followed by 96 trials corresponding to the three characters in their standard versions in the three orientations, each used eight times, and the four distractor characters in the three orientations, each used twice. In addition, trials were organized into a surprise block of 782 trials, composed of 14 preliminary trials, followed by 768 trials corresponding to the three characters in the 24 orientations determined by $15^{\circ}$ increments starting at $0^{\circ}$, each shown eight times, and the four distractor characters in the same 24 orientations, each shown twice. The order of the trials following the 14 preliminary trials was determined randomly. Subjects were given a self-timed break every 55 trials in each block.

Subjects were run in a total of five sessions in Condition 0/45/-90/135 and four sessions in Condition 0/105/-150, each session approximately 1-h long. In the first session subjects were first given the training procedure and then run in two practice blocks. In the second and third sessions subjects were run in four practice blocks per session. In the fourth session subjects were run in two practice blocks followed by a surprise block. In the fifth session of Condition 0/45/-90/135 subjects were run in two more surprise blocks.

\section{Results}

In both conditions incorrect responses, preliminary trials, and distractor trials were discarded. The slope for Block 1 of Condition $0 / 45 /-90 / 135$ was $3.62 \mathrm{~ms} / \mathrm{deg}(276 \mathrm{deg} / \mathrm{s}$ ) (see Fig. 5 b); the slope for Block 1 of Condition 0/105/-150 was $2.59 \mathrm{~ms} / \mathrm{deg}\left(386 \mathrm{deg} / \mathrm{sec}\right.$ ) (see Fig. 7). ${ }^{3}$ These slopes are close to the range of previous estimates of the rate of mental rotation.

In Condition 0/45/-90/135 the slope decreased with each successive block, with Block 12 having a slope of $1.04 \mathrm{~ms} / \mathrm{deg}(962 \mathrm{deg} / \mathrm{s}$ ) (sèe Figs.

${ }^{3}$ These findings replicated the results of a pilot experiment which was identical to Block 1 of Condition 0/45/-90/135. 
a)

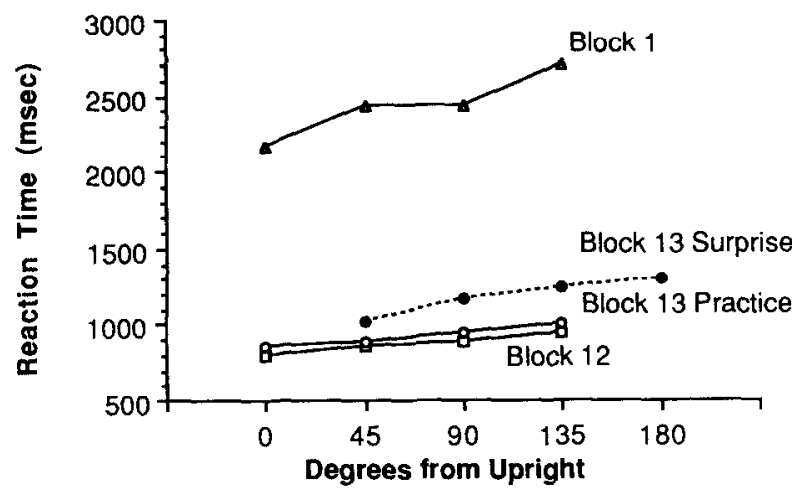

b)

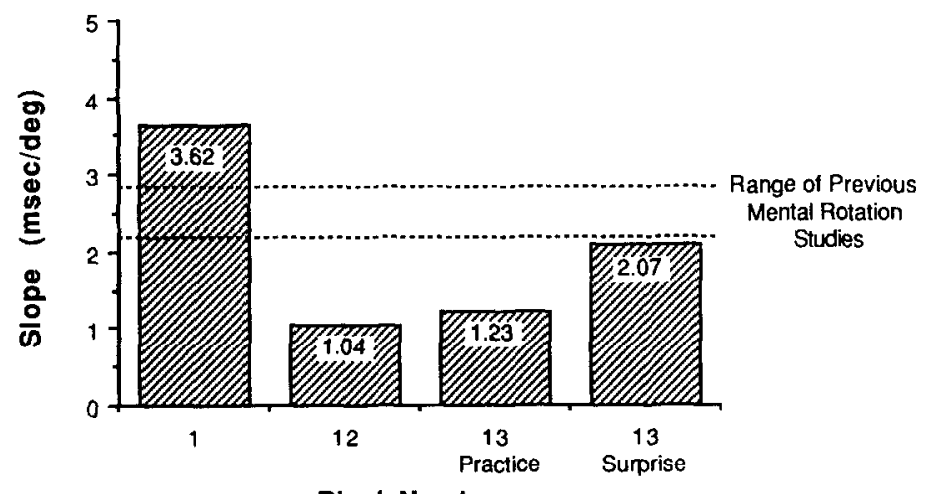

Block Number

Fig. 5. (a) Mean reaction times for recognition as a function of orientation in Blocks 1,12 , and 13 of Condition 0/45/-90/135 of Experiment 2. (b) Slopes for Blocks 1, 12, and 13 of Condition 0/45/-90/135 of Experiment 2.

$5 a$ and $5 b)$. In addition, practice produced an overall decrease in recognition times across all orientations. These effects of practice were analyzed in a two-way ANOVA on data from all practice blocks (1-12) with Block Number and Orientation as factors. As in Experiment 1, a significant main effect for Block $(F(11,121)=27.02, p<.001)$ was found, as well as a significant main effect for Orientation $(F(3,33)=21.05, p<$ .001). A significant interaction between Block Number and Orientation $(F(33,363)=1.86, p<.003)$ revealed that the effect of orientation changed significantly with practice and, as mentioned, was reflected in the data as a decrease in slope with practice. A two-way ANOVA excluding data from $0^{\circ}$ and $180^{\circ}$ revealed no significant interaction between the Block 1 vs Block 13-Surprise factor and the Orientation factor, con- 
sistent with the suggestion that the effects of practice did not transfer to new orientations.

The same pattern occurred in Condition 0/105/-150. The slope decreased with each successive block, culminating with a slope in Block 12 of $0.49 \mathrm{~ms} / \mathrm{deg}$ ( $2041 \mathrm{deg} / \mathrm{s}$ ) (see Fig. 7). In addition, practice produced an overall decrease in recognition times across all orientations. As before, there were significant effects for Block $(F(11,121)=37.48, p<.001)$; Orientation $(F(2,22)=15.41, p<.001)$; and their interaction $(F(22,242)$ $=2.60, p<.001)$.

In Block 13 of Condition 0/45/-90/135, the slope for the practice orientations remained at a low level of $1.23 \mathrm{~ms} / \mathrm{deg}(813 \mathrm{deg} / \mathrm{s})$, while the slope for the same characters at the surprise orientations was $2.07 \mathrm{~ms} / \mathrm{deg}$ $(483 \mathrm{deg} / \mathrm{s})$ (Fig. 5b). By Block 15 the slope for surprise orientations had decreased to $1.05 \mathrm{~ms} / \mathrm{deg}(952 \mathrm{deg} / \mathrm{s})$, close to the $0.87 \mathrm{~ms} / \mathrm{deg}(1149 \mathrm{deg} / \mathrm{s})$ slope obtained for the practice orientations. In Block 13, excluding orientations of $0^{\circ}$ and $180^{\circ}$, there was a significant main effect of Practice versus Surprise orientations $(F(1,11)=32.02, p<.005)$; as expected there was also a main effect of orientation $(F(2,22)=14.60, p<.005)$. There was also a significant interaction between the Practice/Surprise factor and the linear trend of Orientation $(F(1,11)=5.47, p<0.04)$.

Comparable results for Block 13 were found in Condition $0 / 105 /-150$. The slope for the practice orientations was a virtually flat $-0.22 \mathrm{~ms} / \mathrm{deg}$ (Figs. 6 and 7). Because there are several well-learned orientations that subjects might rotate an input shape to, one would not expect recognition times in Block 13 to be monotonically related to orientation, and thus a slope for surprise orientations cannot be measured directly. The pattern of reaction times across orientations is shown in Fig. 6. Unlike the reaction time curves found in Condition 0/45/-90/135 and other recognition studies (Jolicoeur, 1985), the curve for surprise orientations does not increase steadily from $0^{\circ}$ to $180^{\circ}$. Rather it exhibits two components. Over the range of orientation differences from $15^{\circ}$ to $45^{\circ}$ and from $+135^{\circ}$ through $0^{\circ}$ to $-15^{\circ}\left(345^{\circ}\right)$, recognition times for surprise orientations generally increase with distance from the nearest practice orientation, displaying minima at $30^{\circ}$ (near the practice orientation of $0^{\circ}$ ), $+135^{\circ}$ (near the practice orientation of $+105^{\circ}$ ), and at the practice orientation of $210^{\circ}$. However, four orientations clearly do not follow this pattern. For the range of $+60^{\circ}$ to $+120^{\circ}$, response times increase monotonically, even though the practice orientation of $+105^{\circ}$ within this range should have produced a V-shaped function if it attracted rotations to it. Instead, shapes at these orientations appear to have been rotated to the practice orientation at the upright, for reasons that are not clear.

To estimate a rate of rotation for the reaction time data from Condition $0 / 105 /-150$, the orientation difference between each surprise orientation 


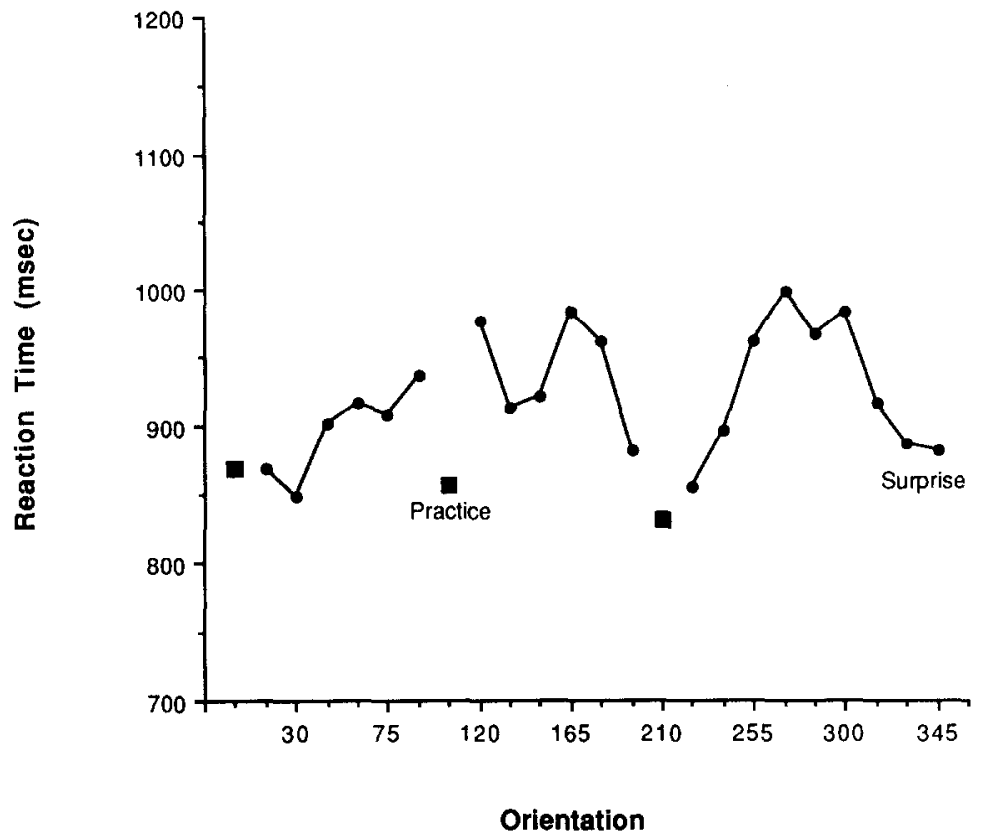

Fig. 6. Mean reaction times for recognition as a function of Practice and Surprise orientations in Block 13 (768 trials) of Condition 0/105/-150 of Experiment 2.

and the closest practice orientation was computed. Averaging across means for orientations at equal distances from a practice orientation, this yields a slope of $1.47 \mathrm{~ms} / \mathrm{deg}(680 \mathrm{deg} / \mathrm{s} ; \mathrm{Fig}$. 7). The correlation between mean reaction time and degrees from the nearest practiced orientation is .88 (Fig. 6); this high correlation occurred despite the anomalous orientations mentioned above where subjects seemed to rotate to the upright in spite of a nearer practice orientation. (The correlation between the distance from the upright and distance from the nearest practice orientation for this set of practice orientations is -0.03 .) Although the estimate of the rate of mental rotation appears lower than expected for a mental rotation function, the discrepancy may be explained by the fact that the surprise block in this experiment presented subjects with a very large number of trials, 768 trials in all. This is far more practice than subjects had in Block 1. It is possible that with this much practice, new orientation-specific representations for surprise orientations were stored and used for recognition before the completion of Block 13. This was not as evident in Condition 0/45/-90/135, because its surprise blocks consisted of only 256 trials. In fact in Condition 0/45/-90/135 one can see the effects of orientation diminishing over the course of the surprise trials: by Block 15, at 


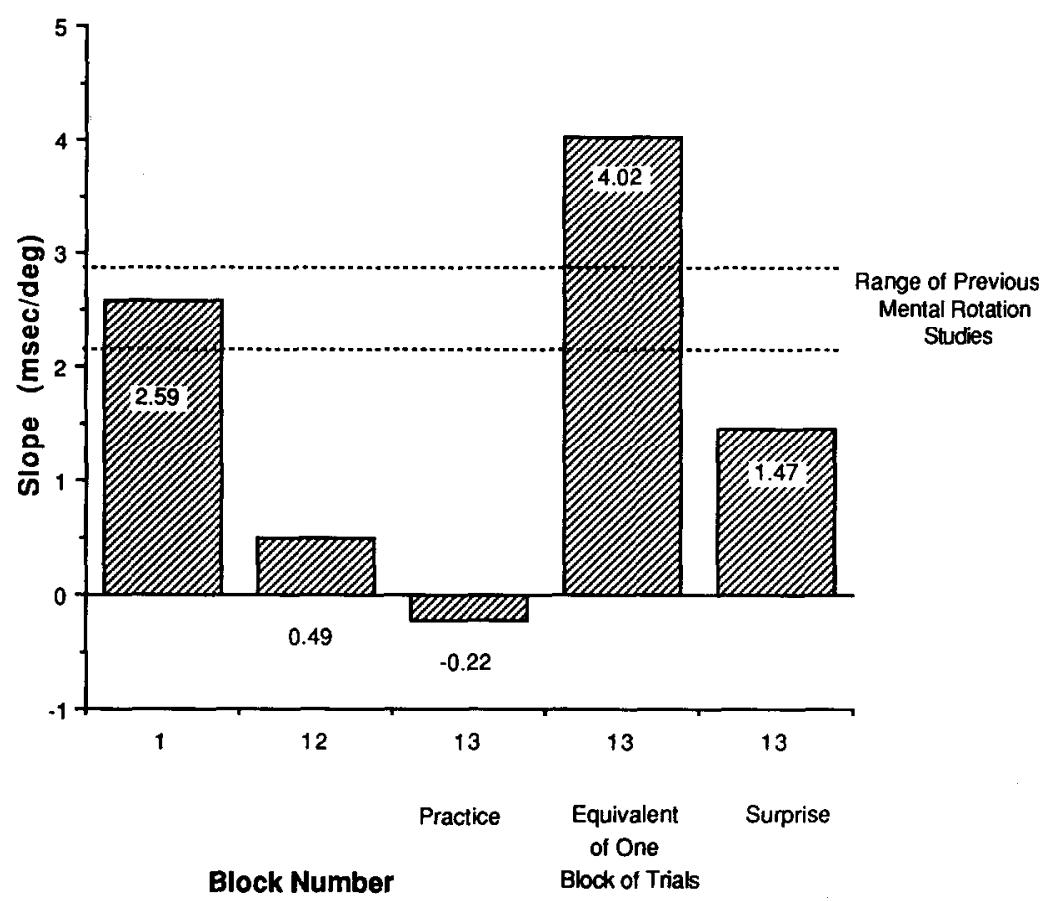

FIG. 7. Slopes for selected blocks of Condition 0/105/-150 of Experiment 2.

which point subjects had gone through a total of 768 trials over three surprise blocks, the slope fell to $1.05 \mathrm{~ms} / \mathrm{deg}(952 \mathrm{deg} / \mathrm{s})$. To estimate the orientation-dependence of shape recognition at surprise orientations before too much practice within the surprise block had accumulated, we analyzed separately the first $5 \%$ of the trials in Block 13 of Condition $0 / 105 /-150$. These trials yielded a slope of $4.59 \mathrm{~ms} / \mathrm{deg}(218 \mathrm{deg} / \mathrm{s})$. The first 96 trials of Block 13, the equivalent of one practice block, were also analyzed separately. A slope of $4.02 \mathrm{~ms} / \mathrm{deg}(249 \mathrm{deg} / \mathrm{s} ; r=.89)$ was found for surprise orientations in the first 96 trials. Both of these slopes are within the range of rotation rates expected from the use of mental rotation. In contrast, a slope of $0.78 \mathrm{~ms} / \mathrm{deg}(1282 \mathrm{deg} / \mathrm{s})$ was found for practice orientations in the first 96 trials; a hypothetical rate of rotation generally considered to be too high to reflect a mental rotation process.

Error rates ranged from about 5-15\% in Block 1 and from about 1-3\% in Block 13. No evidence for a speed/accuracy tradeoff in recognition was found in any condition.

\section{Discussion}

The results are consistent with the hypothesis that mental rotation can 
be used in the recognition of unfamiliar shapes. In both conditions the magnitude of the slope of the RT-orientation function in Block 1 is comparable to the slope obtained in the first block of Experiment 1 and to the slopes obtained in other handedness discrimination studies. This transformation occurred despite the absence of any need to discriminate handedness, either as part of the task instructions or as a consequence of different characters being mirror images of each other (as in the English characters " $p$ " and " $q$ " or " $b$ " and "d"; see Corballis \& McLaren, 1984). This result contradicts any claim that mental rotation is used solely for the determination of handedness, never for shape recognition itself.

Likewise, in both conditions, the effect of orientation diminished, and by Block 12 subjects were recognizing the characters largely independently of their orientation. This suggests that with practice, representations are stored that allow recognition without transformations, either a single orientation-invariant structure or multiple orientation-specific structures. In Block 13 stimuli at practice orientations are still recognized by matching input shapes directly against stored representations, but shapes at surprise orientations are recognized through the use of mental rotation to align the stimulus character with an appropriate representation, a process identical to that used in Block 1. With additional practice, as in Blocks 14 and 15 of Condition 0/45/-90/135, the slope for the surprise orientations decreases to the level of the practice orientations, as orientation-specific representations were stored for the surprise orientations as well, allowing recognition to proceed without using mental rotation.

In Block 13 of Condition 0/105/-150, we can see that subjects not only stored orientation-specific representations for recognition but they are also usually able to use these non-upright representations as targets for mental rotation of characters at new, never-before-seen orientations. In particular, characters observed in orientations near the practice orientations of $0^{\circ}$ and $-150^{\circ}\left(210^{\circ}\right)$ appear to be rotated to these orientations. It is unclear why the recognition of characters observed in orientations near the practice orientation of $+105^{\circ}$ does not seem to be affected by their proximity to this orientation, but are rotated to the practice orientation at the upright. It seems that the upright orientation is "canonical" and that subjects sometimes rotate to it whenever the magnitude of such rotation is not too large. See Robertson, Palmer, and Gomez (1987) for similar findings.

The finding that recognition can be accomplished by rotation to the nearest well-learned orientation adds a great deal of power to a model invoking orientation-specific representations. As few as four equally spaced representations reduce the maximum transformation in the picture plane required for recognition to $45^{\circ}$ or about $100 \mathrm{~ms}$ of processing time 
(assuming a rate of rotation of between 2.0 and $2.5 \mathrm{~ms} / \mathrm{deg}$ or 400 and 500 $\mathrm{deg} / \mathrm{s}$ ), generally less than $15 \%$ of the total time required for recognition (assuming recognition takes about $800 \mathrm{~ms}$ ).

The results of this experiment, then, are consistent with a model of object recognition in which multiple orientation-specific representations are stored, and in which mental rotation can be used to align objects at non-stored orientations with them. It is unlikely that any model that argues for exclusive use of an orientation-invariant representation of objects, such as a structural description expressed in object-centered coordinates, can account for the finding of orientation-dependence in recognition, comparable to effects found in mental rotation studies, for unfamiliar shapes and for familiar shapes at unfamiliar orientations.

\section{EXPERIMENT 3}

Are there any plausible alternative explanations that may account for the findings in Experiment 2? Perhaps we have not adequately ruled out the possibility that mental rotation is used only when handedness is at issue. While subjects were not required to encode or discriminate handedness in Experiment 2, it is conceivable that they took it upon themselves to verify the handedness of each character anyway, to identify it as precisely as possible or to guard against the chance that the reversed version would be shown. It is also possible that subjects always try to determine the handedness of letter-like characters out of habit, because the English alphabet contains asymmetrical characters that may only be written in one version, including mirror-image pairs (" $p$ " and " $q$," " $b$ " and " $d$ ") that must be discriminated both in reading and in writing. If so, they would align the character with the upright to match it against a stored upright representation that encoded handedness. One argument against this is the finding in Condition 0/105/-150 of Experiment 2 that rotations may be to the nearest practice orientation and not to the upright. This result provides evidence that mental rotation in recognition tasks is not used to verify handedness surreptitiously, because the motivation for linking handedness-discrimination to mental rotation is that the only accessible internal representation of a given handedness references the perceiver's egocentric left and right sides (Corballis, 1988; Hinton \& Parsons, 1981). Nonetheless, proponents of an "unnecessary-handednessdiscrimination" hypothesis could cite the results of Experiment 1, which provide evidence that orientation-specific representations at orientations other than upright may also be used to determine handedness. Therefore, new data are needed to address the counterexplanation.

In this experiment, subjects practice recognizing characters for which both standard and mirror-reversed versions were given the same name 
and classified as being a single character, though only one version of each character, which we refer to as the "standard," was extensively taught. (This is analogous to learning the label "glove" in connection with a right-hand glove and applying it to left-hand gloves as well.) If determining handedness is the sole reason for the use of mental rotation in recognition, then defining a recognition task in which handedness is irrelevant by definition, informing subjects of that fact, and giving them practice at treating mirror pairs equivalently, should eliminate the temptation to discriminate between versions of a character. Thus mental rotation should not be used in recognition and no large effects of orientation on recognition are expected, as in the results obtained by Corballis et al. (1978). In contrast, even in an experiment in which both versions of an object must be treated as interchangeable, models using orientation-specific representations predict an effect of orientation on recognition times. This prediction holds whether or not these representations encode handedness. In either case recognition is achieved by using mental rotation to align the observed character with the nearest practice orientation.

\section{Method}

Subjects. Twenty-five students from the metropolitan Boston area participated in the experiment for pay: 13 in Condition 0/105/-150 and 12 in Condition 15/120.

Materials. The stimulus items, computer display, stimulus sets, and general experimental conditions were identical to those used in Experiment 2.

Two conditions were employed. In the first, Condition $0 / 105 /-150$, the practice and surprise orientations were identical to those used in Condition 0/105/-150 in Experiment 2. In the second, Condition $15 / 120$, the characters were displayed in 2 practice orientations, at $+15^{\circ}$ and $+120^{\circ}$ clockwise, and in 22 surprise orientations at $15^{\circ}$ steps clockwise from $0^{\circ}$ not including the two practice orientations (see Fig. 2c). In addition, in Condition 15/120 half of the subjects actually received a set of practice orientations the same distance from the upright, but in a counterclockwise direction, that is, with practice orientations at $-15^{\circ}$ and $-120^{\circ}$. New practice orientations in Condition $15 / 120$ were chosen to meet two criteria. First, all of them were oblique; this eliminates the minor concern that in previous studies the character was displayed on the CRT with perfectly straight lines for the upright training orientation and with slightly jagged oblique lines for most of the other orientations. Second, the practice orientations were limited to a smaller range, resulting in a larger continuous range of surprise orientations. This enabled us to examine the recognition strategy employed by subjects at probe orientations far from a stored representation.

Procedure. The procedure was identical to that used in previous experiments, with the addition that during training subjects were shown the reversed versions of the characters as well as the standard versions and told that the name of a character referred to both its standard and reversed versions. However subjects traced and drew named characters only in their standard versions. Note that in Condition $15 / 120$, subjects were trained on drawings of the standard and reversed versions of the characters at $15^{\circ}$ clockwise or counterclockwise from upright.

Design. In Condition 0/105/-150 trials were organized into practice blocks of 110 trials composed of 14 randomly sclected preliminary trials, followed by 96 trials defined by the three characters in their standard and reversed versions in the three orientations shown four 
times, and the four distractor characters in their standard and reversed versions in the three orientations shown once each. In addition, a surprise block of 782 trials was composed of 14 preliminary trials, followed by 768 trials consisting of the three characters in their standard and reversed versions in the 24 orientations defined by $15^{\circ}$ increments starting at $0^{\circ}$, each shown four times, and the four distractor characters in their standard and reversed versions in the same 24 orientations, each shown once. Subjects were given a self-timed break every 55 trials in each block. In Condition 15/120 trials were organized into practice blocks of 140 trials, composed of 12 randomly selected preliminary trials, followed by 128 trials consisting of the three characters in their standard and reversed versions in the two orientations shown eight times each, and the four distractor characters in their standard and reversed versions in the two orientations shown twice. Surprise blocks were analogous to those used in Condition 0/105/-150. Subjects were given a self-timed break every 70 trials in each block. The order of the trials following the preliminary trials was always determined randomly. Subjects were run in a total of four sessions identical to those in Condition 0/105/-150 of Experiment 2.

\section{Results}

As in previous experiments, incorrect responses, preliminary trials, and distractor trials were discarded. Data from subjects in Condition $15 / 120$ who were trained and practiced on counterclockwise orientations were converted to equivalent clockwise orientations by subtracting the counterclockwise orientations from $360^{\circ}$.

In Condition 0/105/-150 the slope for standard versions in Block 1 was $3.65 \mathrm{~ms} / \mathrm{deg}(274 \mathrm{deg} / \mathrm{s})$. Surprisingly, although the rate of rotation for standard versions is well within the range of estimates of the rate of mental rotation, the slope for reversed versions is essentially flat at $\mathbf{- 0 . 5 5}$ $\mathrm{ms} / \mathrm{deg}$, showing no orientation dependency (see Figs. 9 and 10). Virtually identical results were found in Condition 15/120: the rate of rotation for standard versions is well within the range of estimates of the rate of mental rotation $(4.97 \mathrm{~ms} / \mathrm{deg}$ or $201 \mathrm{deg} / \mathrm{s})$, whereas the slope for reversed versions is essentially flat $(0.42 \mathrm{~ms} / \mathrm{deg}$ or $2381 \mathrm{deg} / \mathrm{s}$; see Figs. 12 and 13).

In Condition 0/105/ - 150 the slope for standard versions decreased with each successive block ending with a slope in Block 12 of $0.44 \mathrm{~ms} / \mathrm{deg}$ $(2273 \mathrm{deg} / \mathrm{s})$. The slope for reversed versions remained constant through all practice blocks ending with a slope in Block 12 of $0.58 \mathrm{~ms} / \mathrm{deg}(1724$ deg/s) (see Fig. 9). In addition, practice resulted in an overall decrease in recognition times across all orientations for both standard and reversed versions. For Blocks 1-12, significant main effects for Block Number were found for both reversed $(F(11,132)=46.88, p<.001)$ and standard $(F(11,132)=42.93, p<.001)$ versions. There were significant main effects of Orientation for standard versions $(F(2,24)=22.11, p<.001)$ and for reversed versions as well despite the nearly flat slope $(F(2,24)=4.71$, $p<0.02$ ), although no significant main effect of Orientation was found for reversed versions in Block 1. A significant interaction between Block Number and Orientation for standard versions $(F(22,264)=4.35, p<$ 
$.001)$ and for reversed versions $(F(22,264)=1.63, p<0.04)$ revealed that the effect of Orientation for both versions changed significantly with practice, although this was reflected in the data as a decrease in slope with practice only for standard versions.

These practice effects are replicated in Condition 15/120, where the slope for standard versions decreased with each successive block ending with a slope in Block 12 of $0.38 \mathrm{~ms} / \mathrm{deg}(2632 \mathrm{deg} / \mathrm{s})$. The slope for reversed versions remained constant through all practice blocks, ending with a slope in Block 12 of $0.57 \mathrm{~ms} / \mathrm{deg}$ (1754 deg/s) (see Fig. 12). In addition, practice resulted in an overall decrease in recognition times across all orientations for both standard and reversed versions. For Blocks 1-12, we found significant main effects for Block Number in both reversed $(F(11,121)=20.84, p<.001)$ and standard $(F(11,121)=18.92$, $p<.001)$ versions. There were significant main effects of Orientation for standard versions $(F(1,11)=9.72, p<0.01)$ and for reversed versions as well $(F(1,11)=34.92, p<.001)$, even though the effects of Orientation in the case of the reversed versions were quite small in magnitude. A significant interaction between Block Number and Orientation for standard versions $(F(11,121)=2.32, p<0.02)$ revealed that the effect of Orientation changed significantly with practice. No such interaction was found for reversed versions. This was reflected in the data as a decrease in slope with practice for standard versions, but no decrease in slope for reversed versions.

A two-way ANOVA on data from Block 1 of Condition 0/105/-150 revealed a significant main effect for Orientation $(F(2,22)=4.20, p<$ $0.05)$, no significant main effect for Version $(F<1)$, and a significant interaction between Orientation and Version $(F(2,22)=5.77, p<0.01)$. A comparable ANOVA on data from Block 1 of Condition 15/120 revealed a significant main effect for Orientation $(F(1,11)=15.56, p<.002)$, no significant main effect for Version, and no significant interaction between Orientation and Version.

In Block 13 of Condition 0/105/ - 150 the slope for the practice orientations was $0.46 \mathrm{~ms} / \mathrm{deg}(2174 \mathrm{deg} / \mathrm{s})$ for reversed versions and 0.69 $\mathrm{ms} / \mathrm{deg}(1449 \mathrm{deg} / \mathrm{sec})$ for standard versions. Figure 8 shows that, as in Experiment 2, over most but not all of the range of orientations, the recognition times are consistent with a strategy of rotation to the nearest stored orientation. Specifically, response times increase as a function of distance from the $0^{\circ}$ practice orientation over the range from $+15^{\circ}$ to $+45^{\circ}$ and from $-15^{\circ}\left(345^{\circ}\right)$ to $-75^{\circ}\left(285^{\circ}\right)$ (standard) or $-90^{\circ}\left(270^{\circ}\right)$ (reversed). Similarly response times increased monotonically with distance from the $-150^{\circ}\left(210^{\circ}\right)$ practice orientation from $-135^{\circ}\left(225^{\circ}\right)$ to $-75^{\circ}\left(285^{\circ}\right)$ (standard) or $-90^{\circ}\left(270^{\circ}\right)$ (reversed) and from $-165^{\circ}\left(195^{\circ}\right)$ to $180^{\circ}$. Stimuli at $+120^{\circ}$ and $+135^{\circ}$ also appear as if they have been rotated 


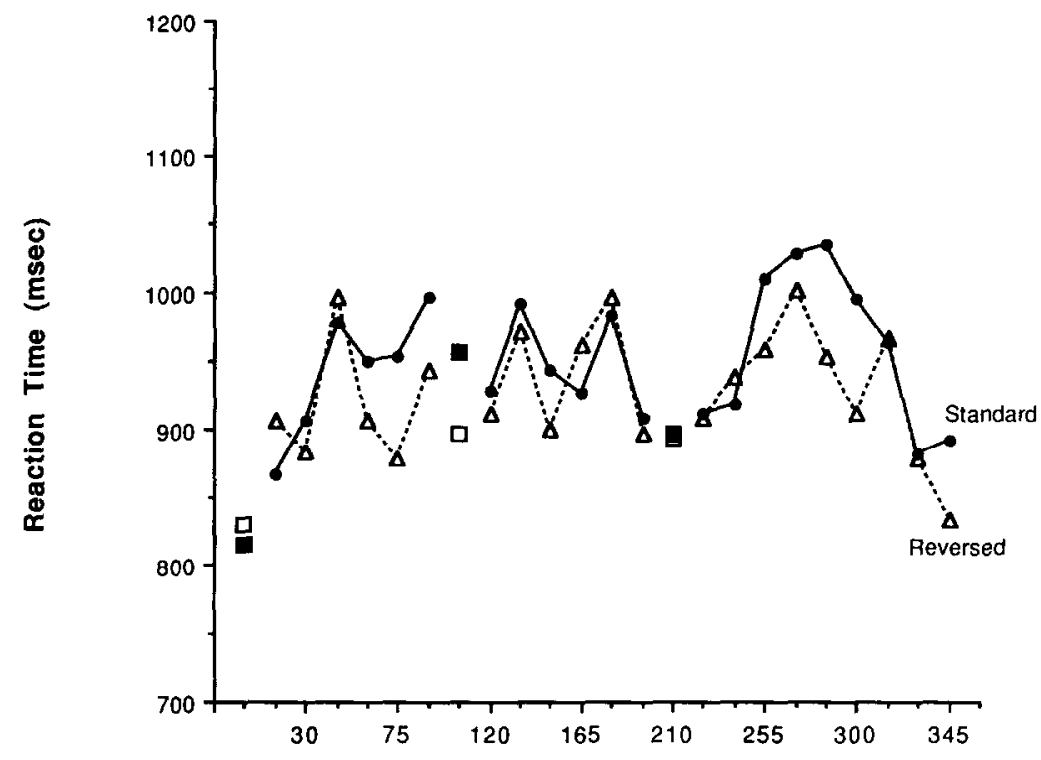

Orientation

Fig. 8. Mean reaction times for recognition of standard and reversed versions as a function of Practice and Surprise orientations in Block 13 (768 trials) of Condition 0/105/-150 of Experiment 3.

to the practice orientation at $+105^{\circ}$. However, the three data points in the range from $+60^{\circ}$ to $+90^{\circ}$ and those at $+150^{\circ}$ and $+165^{\circ}$ are anomalous, reflecting rotation neither to the upright nor to the nearest stored orientation.

Despite these anomalies, very clear overall effects of rotation to the nearest stored orientation can be measured. When response times are regressed against the orientation difference between a surprise stimulus and the closest practice orientation, one obtains a slope of $2.06 \mathrm{~ms} / \mathrm{deg}$ $(485 \mathrm{deg} / \mathrm{s} ; r=.99)$ for standard versions and $0.92 \mathrm{~ms} / \mathrm{deg}(1087 \mathrm{deg} / \mathrm{s} ; r$ $=.92$ ) for reversed versions. Again, we sought cleaner estimates of possible rotation rates, prior to the learning of individual surprise orientations over the course of the lengthy surprise block. For the first $5 \%$ of the trials in Block 13, one obtains a slope of $11.23 \mathrm{~ms} / \mathrm{deg}(89 \mathrm{deg} / \mathrm{s})$ for standard versions and $3.63 \mathrm{~ms} / \mathrm{deg}(275 \mathrm{deg} / \mathrm{s})$ for reversed versions. This unusually slow rate of rotation for standard versions is due to a single subject. A second estimate of the initial rotation rate in the surprise block was calculated by examining the first 96 trials, the equivalent of one practice block. For surprise orientations this yielded slopes of $4.14 \mathrm{~ms} / \mathrm{deg}(242$ 
$\mathrm{deg} / \mathrm{s} ; r=.87)$ for standard versions and $3.36 \mathrm{~ms} / \mathrm{deg}(298 \mathrm{deg} / \mathrm{s} ; r=.79)$ for reversed versions. These slopes, with the exception of those influenced by the slow subject, are within the range of estimates of mental rotation rates (see Fig. 9). In contrast, at practice orientations in the first 96 trials, a slope of $0.36 \mathrm{~ms} / \mathrm{deg}(2778 \mathrm{deg} / \mathrm{s})$ was found for standard versions and $0.09 \mathrm{~ms} / \mathrm{deg}(11111 \mathrm{deg} / \mathrm{s})$ for reversed versions; both putative rates of rotation are generally considered to be too fast to be attributed to a mental rotation process.

Similar results were found in Block 13 of Condition 15/120. The slope for the practice orientations was $0.45 \mathrm{~ms} / \mathrm{deg}(2222 \mathrm{deg} / \mathrm{s})$ for reversed versions and $1.12 \mathrm{~ms} / \mathrm{deg}(893 \mathrm{deg} / \mathrm{s})$ for standard versions. The pattern of reaction times, shown in Fig. 11, is partially consistent with rotation to the nearest practice orientation for both standard and reversed versions. Roughly, response times increase with distance from the nearest practice orientation, though the maxima are displaced somewhat from the locations we would expect if the "attraction" of each stored representation was equal in all directions: $90^{\circ}$ (standard) rather than $60^{\circ}-75^{\circ}$ (the maximum for the mirror-reversed stimuli was $75^{\circ}$ ) and $270^{\circ}$ rather than $240^{\circ}$. In

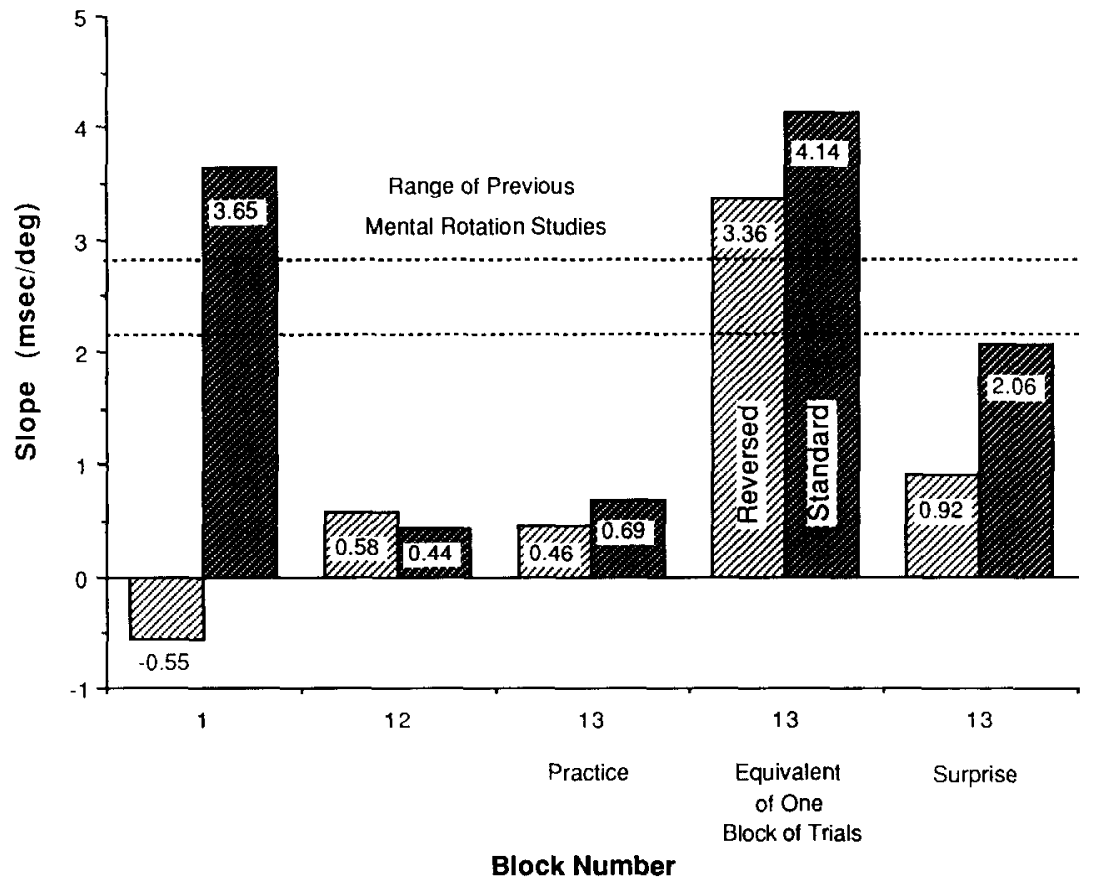

FIG. 9. Slopes for standard and reversed versions of characters for selected blocks of Condition 0/105/-150 of Experiment 3. 


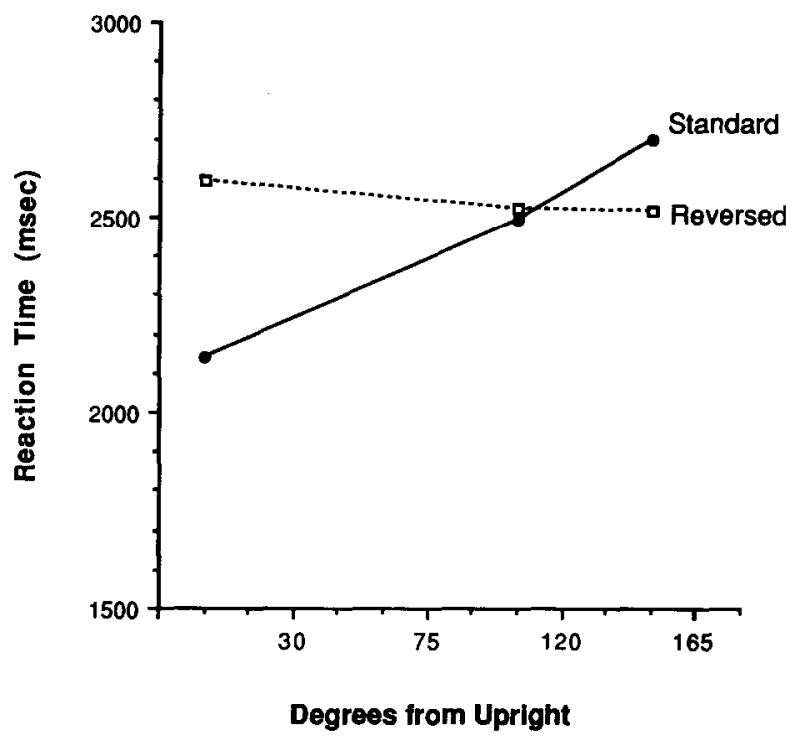

FIG. 10. Mean reaction times for recognition of standard and reversed versions as a function of orientation in Block 1 of Condition 0/105/-150 of Experiment 3.

addition, the response time function was not perfectly monotonic within these ranges, but displayed some noise. Nonetheless, there were clear measurable effects of distance to the nearest practice orientation. Rotation rates were estimated by regressing the response times against the difference between each surprise orientation and the practice orientation that was nearest to it. This analysis yielded a slope of $1.13 \mathrm{~ms} / \mathrm{deg}(885$ $\mathrm{deg} / \mathrm{s} ; r=.85)$ for standard versions and $0.71 \mathrm{~ms} / \mathrm{deg}(1408 \mathrm{deg} / \mathrm{s} ; r=.73)$ for reversed versions. (The correlation between the distance from the upright and distance from the nearest practice orientation for this set of practice orientations is -0.34 .) The first $5 \%$ of the trials in Block 13 were analyzed separately and yielded slopes comparable to those obtained in previous mental rotation studies: $3.26 \mathrm{~ms} / \mathrm{deg}(307 \mathrm{deg} / \mathrm{s} ; r=.54)$ for standard versions in surprise orientations and $2.05 \mathrm{~ms} / \mathrm{deg}(488 \mathrm{deg} / \mathrm{s} ; r=$ $.53)$ for reversed versions in surprise orientations (see Fig. 12). In contrast, at practice orientations in the first $5 \%$ of the trials, where we do not expect much rotation to occur, a slope of $2.77 \mathrm{~ms} / \mathrm{deg}(361 \mathrm{deg} / \mathrm{s})$ was found for standard versions and $-0.70 \mathrm{~ms} / \mathrm{deg}(-1429 \mathrm{deg} / \mathrm{s})$ for reversed versions; the former rate is within the range of rotation rate estimates but still faster than that obtained for surprise orientations. The first $\mathbf{1 2 8}$ trials of Block 13, the equivalent of one practice block, also were analyzed separately. For the first 128 trials slopes of $1.86 \mathrm{~ms} / \mathrm{deg}(538 \mathrm{deg} / \mathrm{s})$ for standard versions and $1.35 \mathrm{~ms} / \mathrm{deg}(741 \mathrm{deg} / \mathrm{s})$ for reversed versions were obtained. 


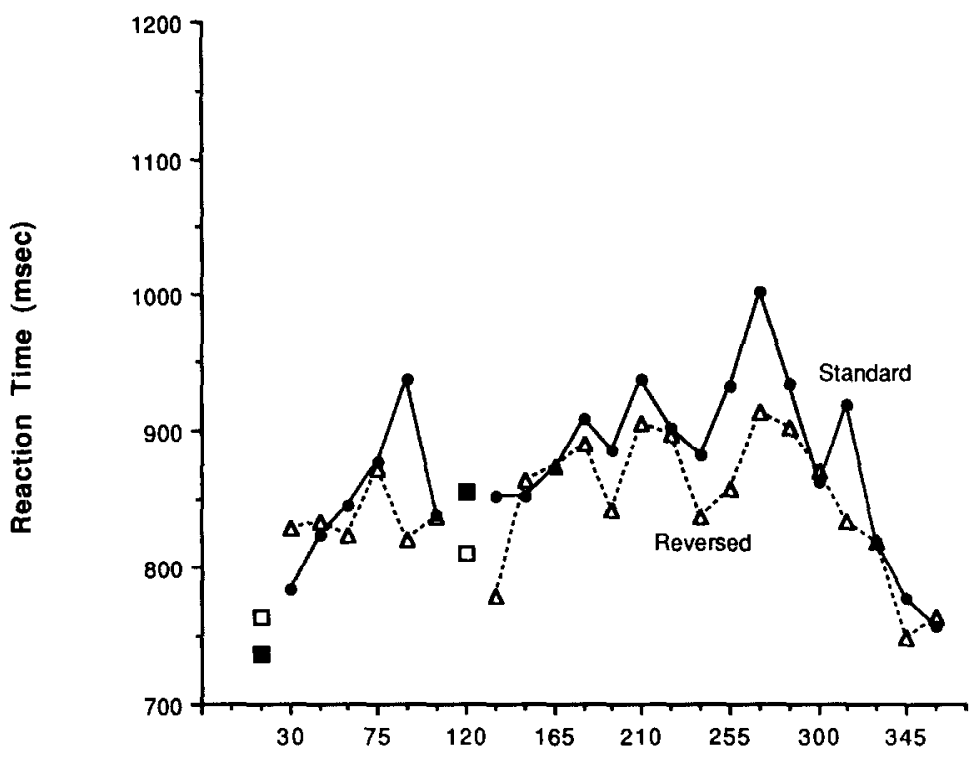

Orientation

Fig. 11. Mean reaction times for recognition of standard and reversed versions as a function of Practice and Surprise orientations (with counterclockwise practice and surprise orientations converted to clockwise) in Block 13 (768 trials) of Condition 15/120 of Experiment 3.

Error rates ranged from about $4-16 \%$ in Block 1 and from about $0.5-5 \%$ in Block 13. No speed/accuracy tradeoffs were found in any of the conditions.

\section{Discussion}

Despite the fact that subjects were required to treat both normal and mirror-reversed versions of each character equivalently in this experiment, the effects of orientation on the normal versions of each character did not differ significantly from the effects of orientation in Experiment 2. In addition, in both conditions the recognition of both standard and reversed versions is orientation-dependent in the early portion of Block 13 for surprise orientations. In each of these instances, the rate of rotation is comparable to the rate expected from the use of mental rotation in previous studies. These results make it unlikely that subjects performed these transformations because of their spontaneous or habit-driven adoption of a strategy whereby they would determine the handedness of a shape even when not required to do so; in this experiment the task demands specifically required them to ignore handedness. Moreover, the 


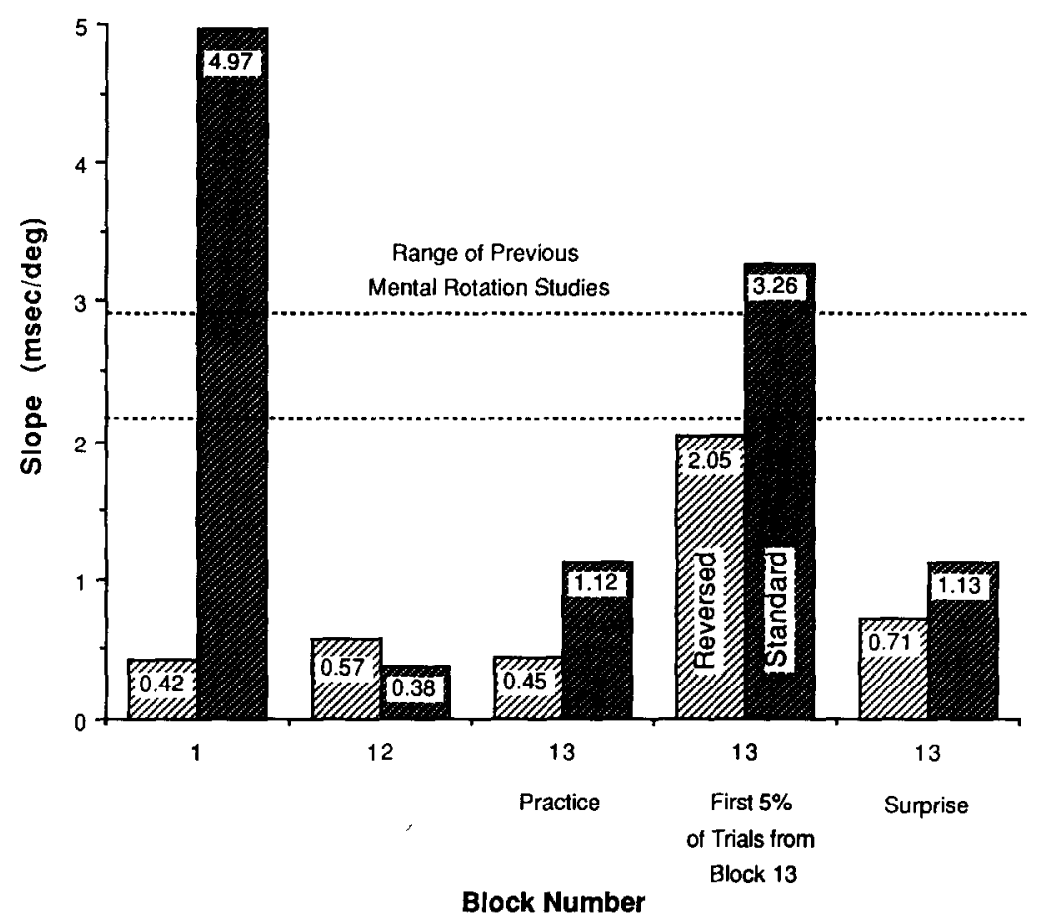

FIG. 12. Slopes for standard and reversed versions of characters for selected blocks of Condition 15/120 of Experiment 3.

results are compatible with our previous findings on the use of orientation-specific representations in recognition and on the use of mental rotation on characters in unstored orientations, usually to align them with the nearest stored representations. In particular, characters observed in surprise orientations near the practice orientations, with some exceptions, appear to be rotated to the nearest practice orientation. Curiously, in Condition $0 / 105 /-150$ the data also suggest a special status for the surprise orientation of $+150^{\circ}$ for mirror-reversed characters and $+165^{\circ}$ for standard characters. These orientations act as if they were practice orientations to which characters at other surprise orientations were rotated. A similar result for the anomalous surprise orientation of $-120^{\circ}$ $\left(240^{\circ}\right)$ in Condition $15 / 120$ conceivably might be accounted for by a flip about the vertical axis to align the figure with the practice orientation at $+120^{\circ}$; see Corballis et al. (1978) for a similar result and possible explanation.

This experiment also yielded an unexpected finding: shapes corresponding to the mirror-images of learned but unpracticed characters are not recognized more slowly at orientations further from the upright. This 


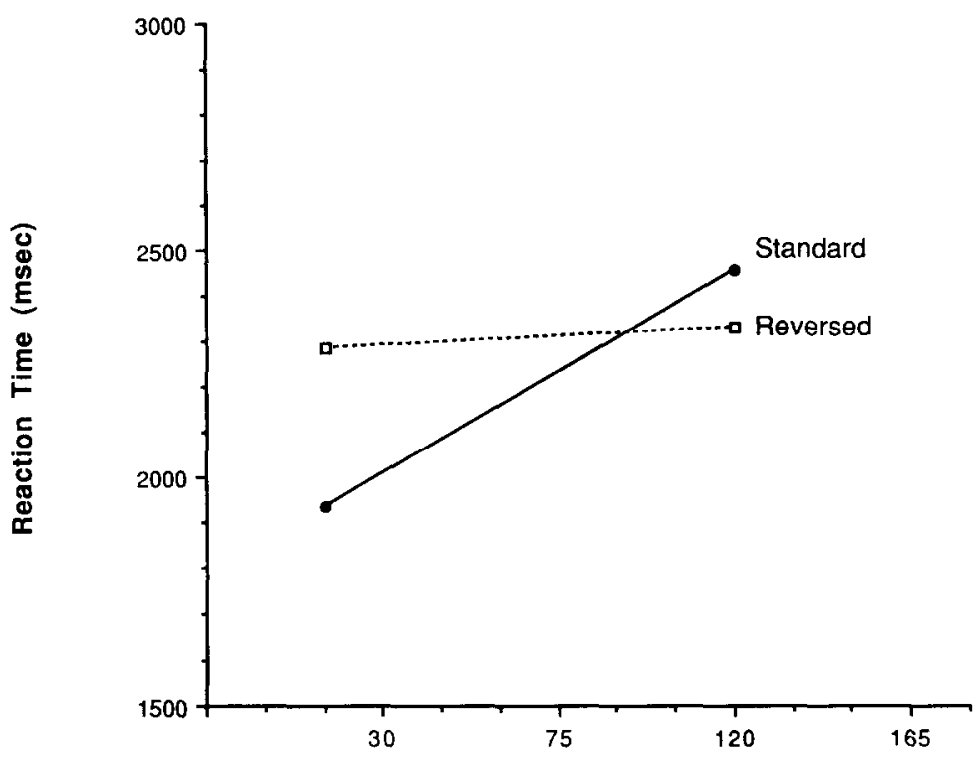

Degrees from Upright

FIG. 13. Mean reaction times for recognition of standard and reversed versions as a function of orientation in Block 1 of Condition 15/120 of Experiment 3.

provides further evidence for the handedness-specificity of the representations used for recognition. The representation to which the standard version was rotated in the first block appears not to have triggered an analogous picture-plane rotation of mirror-reversed versions of the same shapes to the upright, suggesting that it was in a form that was specific to the standard handedness. Presumably this was a consequence of the training procedure, in which subjects were shown both versions of shapes, but only tried tracing and drawing the standard versions, leading them to store representations of the standard but not the reversed versions at the upright orientation. This was tested in a distinct control condition in which 14 subjects were initially required to learn both the standard and mirror-reversed versions of each shape prior to testing. When tested in the equivalent of Block 1 of Condition $0 / 105 /-150,{ }^{4}$ they showed equivalent orientation effects for normal and mirror-reversed versions, as predicted (see Fig. 14). Specifically, the slope for standard versions of the characters was $2.27 \mathrm{~ms} / \mathrm{deg}$ ( $441 \mathrm{deg} / \mathrm{s}$ ), while the slope for reversed versions of the characters was $1.36 \mathrm{~ms} / \mathrm{deg}(735 \mathrm{deg} / \mathrm{s})$; there was a significant

\footnotetext{
${ }^{4}$ The experimental apparatus was the same as that used in Experiment 4.
} 


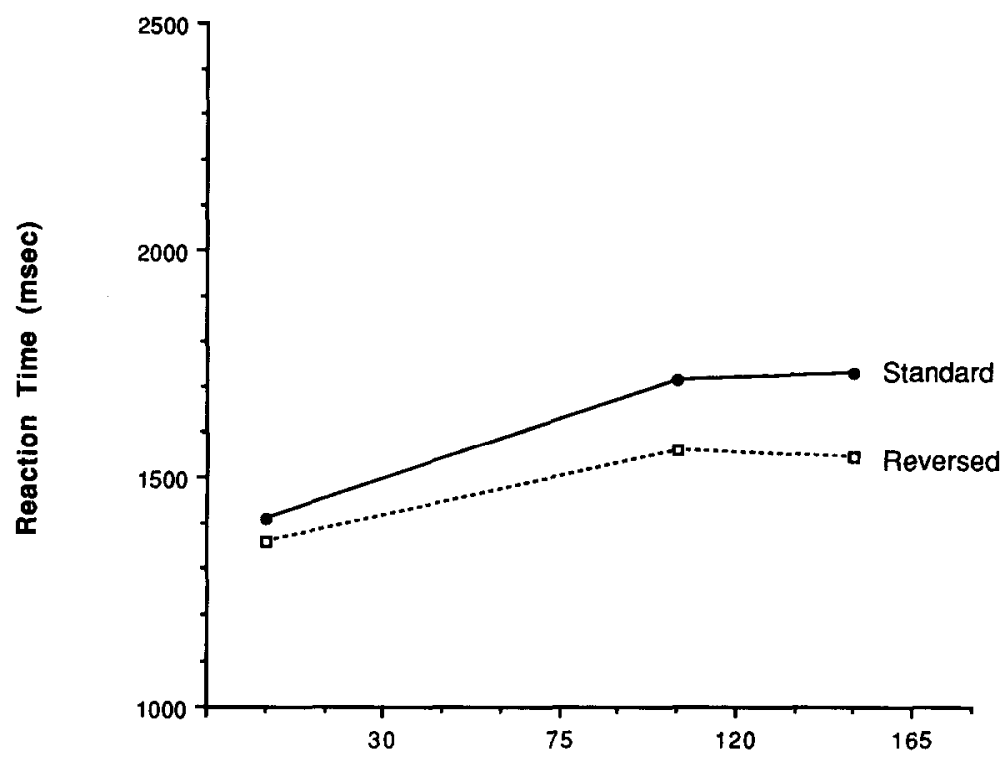

Degrees from Upright

FIG. 14. Mean reaction times for recognition of standard and reversed versions as a function of orientation in the Control Condition of Experiment 3.

main effect for Orientation $(F(2,26)=11.36, p<.001)$, a significant main effect for Version $(F(1,13)=9.48, p<0.01)$, and no significant interaction $(F<1)$.

Notc also that the difference in response patterns for normal and mirror-reversed shapes provides additional evidence against the possibility that subjects are rotating merely to assess handedness. If they were doing that, we would expect them to show the same pattern of data as the subjects in Experiment 1, where assessing handedness was exactly what the subjects were required to do. However, subjects in Experiment 1 were orientation-sensitive for both standard and reversed versions shapes to the upright, unlike the subjects in the present experiment.

Why did subjects take equal amounts of time for all orientations for the mirror-reversed versions of the shapes they learned? The simplest explanation is that they mentally flipped the input shapes in depth through the shortest path by which the shapes would match their counterparts in memory. Parsons (1987a, b) shows that for a two-dimensional shape and its mirror-image, both in the picture plane, this shortest path corresponds to a rotation about an axis in the picture plane, different for each pictureplane orientation difference, and that the amount of rotation is always 
$180^{\circ}$. Thus if subjects adopted a consistent strategy of mentally rotating input shapes along the shortest path that would align them with an upright memorized standard, they would automatically rotate standard versions of shapes within the picture plane around the line of sight axis by an amount that would depend on the shape's misorientation, and would rotate mirror-reversed versions in depth around an axis in the picture plane that would depend on the shape's misorientation by a constant amount $\left(180^{\circ}\right)$, which corresponds to the interaction found in the data. The fact that the two curves in Fig. 10 and in Fig. 13 cross, rather than converge at $180^{\circ}$, could reflect the rotation process (rate, intercept, or both) being faster around picture-plane axes than around the line-of-sight axis. Parsons $(1987 \mathrm{c})$ has gathered evidence that under certain circumstances this is the case.

Parsons $(1987 \mathrm{a}, \mathrm{b})$ invoked shortest-path rotation (among other mechanisms) in accounting for a similar pattern of data he found in several experiments. In Parsons (1987a), subjects judged whether a line drawing of a person had its right or left arm raised, a task that induces subjects to imagine themselves moving into the depicted orientation. For views of backs of bodies, which correspond to the subject's egocentric reference frame, response time increased with orientation from the upright; for views of fronts of bodies, which are flipped with respect to the subjects' reference frame, response times were virtually independent of pictureplane orientation. Similar interactions were found in three experiments in Parsons (1987b), where subjects imagined their hands moving into the orientation of a misoriented hand depicted either palm-down (resulting in response times increasing monotonically with misorientation) or palm-up (resulting in response times showing no such increase).

Why does orientation-sensitivity return for reversed versions in surprise orientations in Block 13? Presumably, subjects learned representations for the reversed shapes at the practice orientations in Blocks 1 through 12, causing the shortest path of rotation now to be within the picture-plane to a representation of the reversed version, rather than a flip in depth to a representation of the standard version. This is supported by the results of the control condition, where subjects explicitly learned the mirror version of the shapes prior to the recognition task, and immediately rotated misoriented examplars within the picture plane to the learned upright orientation.

There are two kinds of independent evidence consistent with the suggestion that people find and execute shortest-3D-path rotations. First, data suggestive of this process appear exactly in those tasks in which it would be an efficient strategy. Mirror-images are recognized in constant time in the recognition tasks of Experiments 3 (and as we shall see, in Experiment 4), but not in Experiment 1 and other handedness discrimi- 
nation tasks, because the shortest-3D-path strategy destroys handedness information for two-dimensional shapes and so must be avoided in such tasks. In experiments using the same recognition paradigm as in Experiments 3 and 4, but with three-dimensional shapes, mirror-images (enantiomorphs) of the shapes are not recognized in constant time (Tarr, 1989). This is exactly what one would expect since enantiomorphs cannot be superimposed exactly by any rotation in three dimensions (instead, subjects rotated the reversed shapes into partial correspondence with the practiced orientations of the standard version, through paths of various lengths less than or equal to $180^{\circ}$ ). The use of shortest-path rotation by subjects in Parsons' handedness-discrimination studies (1987a, b) is also consistent with this analysis. His shapes were defined in three dimensions, so flips do not destroy handedness information and subjects had no reason to avoid them. Subjects had available to them two standard representations (one each for their left and right body parts), and the evidence for shortest-path rotations always involved the aligning of two views of the same-side body part, never of a left body part with a right body part.

The second line of support comes from Shepard (1984), who has provided evidence that when people see alternating displays of two views of a shape, they experience apparent motion of the shape along the shortest path, showing that the perceptual system must be able to find such axes. We have created displays in which an upright standard version of one of our shapes alternates with a misoriented mirror-reversed version; the illusion that the shape flips in depth around the appropriate picture-plane axis is compelling and occurs for pairs at all orientation disparities. If Shepard's conjecture is correct that mental rotation and apparent motion involve common mechanisms, this demonstration suggests that shortestpath mental rotations for our shapes can be easily triggered.

\section{EXPERIMENT 4}

We have been assuming that the multiple representations of a shape that people store are simultaneously specific to an orientation and a handedness. This assumption is necessary to explain the fact that Corballis et al. (1978) found effects of orientation in the recognition of mirror-reversed but not standard letters, presumably because subjects possessed multiple orientation-specific version-specific representations of standard English letters and digits and a single representation of reversed English letters and digits at the upright. Similarly, we explained the results of Experiment 3 by claiming that at the beginning of the experiment subjects possessed only a representation of the standard version of the characters at the upright, and by the beginning of the surprise blocks they possessed representations of both versions of the characters at each of the practice 
orientations. However, this assumption has not yet been independently established. To be sure, the results of Experiment 1, in which highly practiced subjects discriminated handedness equally quickly at all and only familiar orientations, show that people can form simultaneous handedness-specific and orientation-specific representations. However, this could be a product of the overt handedness judgment required, and under normal circumstances multiple representations at different orientations do not contain handedness information. Experiments 2 and 3 do not settle the matter either because subjects were only surprised with versions of characters that they had practiced recognizing in early blocks. Therefore the results could not differentiate definitively between the formation of handedness-general and handedness-specific non-upright representations in recognition.

In this experiment subjects received practice only with standard versions of characters and were then tested at surprise orientations with both standard and reversed versions. If the multiple non-upright representations that subjects store are handedness-general, then both standard and reversed versions in surprise orientations will be recognized by rotation to the nearest stored representation. However, if the stored representations are handedness-specific, subjects cannot align reversed versions with them by a rotation in the picture plane, and should show no orientation effects, just as in the first blocks of Experiment 3 (presumably because the shortest-path rotations would be a constant $180^{\circ}$ rotation in depth).

\section{Method}

Subjects. Thirtcen students from the metropolitan Boston area participated in the experiment for pay.

Materials. The stimulus items, orientations, stimulus sets, and general experimental conditions were identical to those used in Condition 15/120 of Experiment 3. However, different hardware was used: the new display monitor had a resolution of $480 \times 320$ pixels, rather than the $320 \times 240$ pixel resolution of the display used in previous experiments, and was controlled by an IBM PC/XT microcomputer. The distance of the display from the subject and the visual angle of characters remained the same as those in previous experiments.

Procedure. The training procedure was identical to that used in Condition 15/120 of Experiment 3, except that subjects saw only the drawings of the standard versions of the characters. Although subjects were never shown the reversed versions, they were informed that the names of the characters applied even if the character was mirror-reversed.

Design. Trials were organized into practice blocks of 134 trials, consisting of 6 randomly selected preliminary trials, followed by 128 trials consisting of the three characters in their standard versions in the two orientations, shown 16 times, and the four distractor characters in their standard versions in the two orientations shown 4 times. Surprise blocks of 768 experimental trials were identical to those used in Experiment 3, preceded by 6 preliminary trials. Subjects were given a self-timed break every 70 trials in each block. Subjects were run in a total of four sessions corresponding to those in Experiment 3. 


\section{Results}

As in previous experiments, incorrect responses, preliminary trials, and distractor trials were discarded, and data from subjects trained and practiced on counterclockwise orientations were converted to equivalent clockwise orientations by subtracting the counterclockwise orientations from $360^{\circ}$. As shown in Fig. 15, the slope in Block 1 was $1.45 \mathrm{~ms} / \mathrm{deg}(690$ $\mathrm{deg} / \mathrm{s})$. Although this slope is lower than that obtained in Block 1 of Experiments 1, 2, and 3, it may be attributable to the small number of orientations and the presence of only standard versions in the practice blocks. Since subjects only needed to store two orientation-specific representations, it is possible that this was accomplished early in Block 1, alleviating the need to use mental rotation in trials at the end of the block. This hypothesis was confirmed by the finding that for the first $5 \%$ of trials in Block 1 the slope was $2.45 \mathrm{~ms} / \mathrm{deg}$ ( $408 \mathrm{deg} / \mathrm{s})$, a rate comparable to the rates of rotation found in previous mental rotation studies.

The slope decreased over blocks, ending with a slope in Block 12 of $0.31 \mathrm{~ms} / \mathrm{deg}(3226 \mathrm{deg} / \mathrm{s})$. In addition, practice resulted in an overall decrease in recognition times across all orientations. Significant main effects were found for Block $(F(11,132)=37.74, p<.001)$ and for Orientation $(F(1,12)=23.54, p<.001)$. A significant interaction between Block Number and Orientation $(F(11,132)=2.44, p<0.01)$ reflected the decrease in slope with practice.

In Block 13 the slope for the practice orientations was $0.81 \mathrm{~ms} / \mathrm{deg}$ $(1235 \mathrm{deg} / \mathrm{s})$ for reversed versions and $0.31 \mathrm{~ms} / \mathrm{deg}(3226 \mathrm{deg} / \mathrm{s})$ for stan-

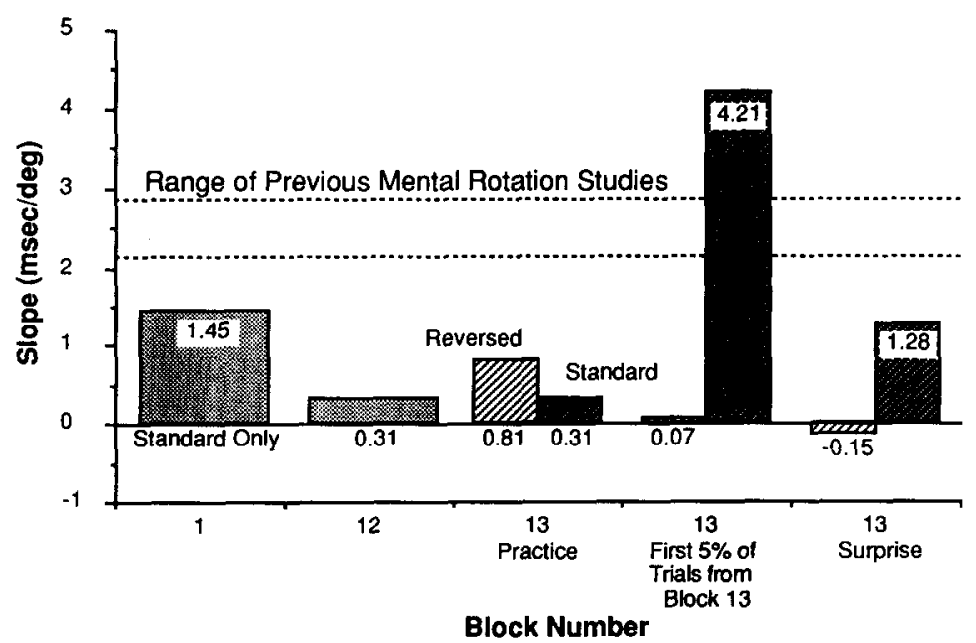

FIG. 15. Slopes for standard versions of characters for selected practice Blocks and for standard and reversed versions of Block 13 of Experiment 4. 
dard versions. For surprise orientations, the pattern of reaction times, shown in Fig. 16, suggests that, as in our previous experiments, standard versions of characters observed at surprise orientations were rotated to the nearest practice orientation for most of the range of orientations. Although the response times in the range from $+30^{\circ}$ to $+105^{\circ}$ are difficult to interpret, the response times in the range from $+135^{\circ}$ through $180^{\circ}$ to $0^{\circ}$ generally increase with distance away from each of the practice orientations bounding this range. In contrast, no orientation dependency is apparent for reversed versions, which appear to have a flat function across all orientations (except for $180^{\circ}$ ). Response times for surprise orientations werc regressed against the rotation angle to the nearest practice orientation; this analysis produced a slope of $1.28 \mathrm{~ms} / \mathrm{deg}(781 \mathrm{deg} / \mathrm{s} ; r=$ .98) for standard versions and $-0.15 \mathrm{~ms} / \mathrm{deg}(r=.26)$ for reversed versions, exactly as expected. For the first $5 \%$ of the trials in Block 13, before additional practice could accumulate, analysis yielded a slope of $4.21 \mathrm{~ms} / \mathrm{deg}(238 \mathrm{deg} / \mathrm{s} ; r=.56)$ for standard versions in surprise orientations (a slope within the range of prior estimates of the rate of mental rotation) and a flat slope of $0.07 \mathrm{~ms} / \mathrm{deg}(14286 \mathrm{deg} / \mathrm{s} ; r=.01)$ for reversed versions in surprise orientations. In contrast, at practice orientations in

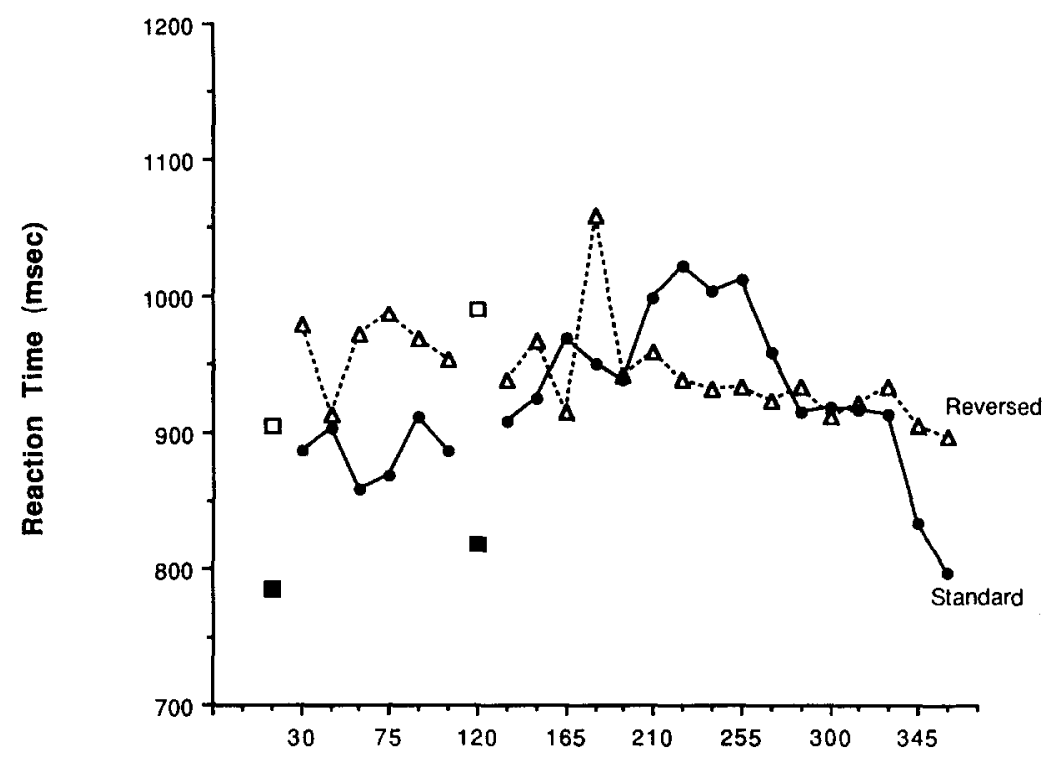

Orientation

FIG. 16. Mean reaction times for recognition of standard and reversed versions as a function of Practice and Surprise orientations (with counterclockwise practice and surprise orientations converted to clockwise) in Block 13 (768 trials) of Experiment 4. 
the first $5 \%$ of the trials, a slope of $0.02 \mathrm{~ms} / \mathrm{deg}(50000 \mathrm{deg} / \mathrm{s})$ was found for standard versions and $1.90 \mathrm{~ms} / \mathrm{deg}(526 \mathrm{deg} / \mathrm{s})$ for reversed versions. The first 128 trials of Block 13, the equivalent of one practice block, also were analyzed separately, yielding slopes of $1.76 \mathrm{~ms} / \mathrm{deg}(568 \mathrm{deg} / \mathrm{s})$ for standard versions and $-0.16 \mathrm{~ms} / \mathrm{deg}$ for reversed versions. Error rates were around $3.5 \%$ in Block 1 and ranged from 0 to $4 \%$ in Block 13 . No speed/accuracy tradeoffs were in evidence.

\section{Discussion}

The results of Experiment 4 support the claim that representations used in recognition, both the canonical upright orientation formed when first learning the character and the set of non-upright representations formed with practice, are simultaneously orientation-specific and handednessspecific. This is revealed by the finding that stored representations at practice orientations enabled picture-plane rotations only for the recognition of the version in which they were originally practiced. No effect of orientation on recognition time was found at any point in Block 13 for reversed versions of the characters displayed in surprise orientations, replicating the results of Block 1 of both conditions of Experiment 3, and further supporting the hypothesis that subjects rotated along the shortest path in three dimensions.

These results also cast further doubt on the hypothesis that representations are orientation-invariant, but without encoding handedness, and that mental rotation is performed in recognition solely to confirm handedness on the chance that handedness matters. This hypothesis predicts that if stored representations facilitate the determination that a stimulus is of standard handedness, they should also facilitate the determination that a stimulus is reversed, which is just the "else" condition or complementary response for the standard-version test. But if this were the case there is no reason why reversed versions in Block 13 would not have been rotated in the picture plane to the nearest practice orientation to discriminate version. Thus, it seems unlikely that in recognition tasks rotations to the nearest practice orientation are performed to confirm or determine handedness. Overall our results have consistently demonstrated an effect of orientation for shapes observed in surprise orientations, supporting the claim that subjects are capable of using orientation-specific representations for the recognition of familiar objects.

\section{GENERAL DISCUSSION}

In these studies, we tested three major theories of shape recognition by having subjects recognize misoriented confusable shapes that they had learned in specific orientations. We found that:

- When subjects first had to recognize misoriented characters, the time 
they required was generally monotonically related to the rotation of the characters from the upright orientation at which they had learned them. This was true despite the fact that no handedness discrimination was required.

- With practice at recognizing the characters in particular orientations, subjects' recognition times became roughly equivalent across all practiced orientations.

- Following training or practice with a character in a standard handedness in particular orientations, recognition times increased with differences in orientation between the stimulus character and a stored orientation, which was most often the nearest well-learned orientation, but sometimes the canonical upright orientation.

- This orientation-dependence can probably be attributed to the use of "mental rotation," because the slope of the recognition time functions, estimating the rate of mental rotation, were consistently close to the slopes found in previous mental rotation studies, including ones that used converging techniques to demonstrate the analog nature of the rotation process, and even closer to the slopes found in Experiment 1, where the same characters were used in a task that uncontroversially requires mental rotation (see Fig. 17).

- When mirror-images of familiar shapes were recognized, response

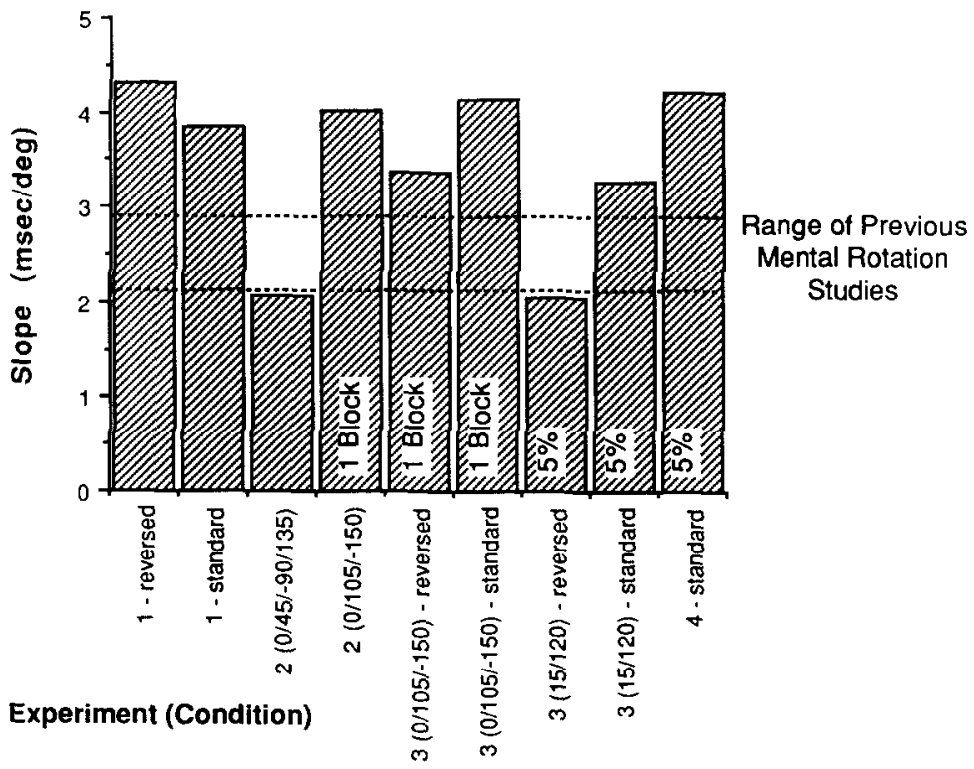

Fig. 17. Summary of slopes for surprise orientations in surprise block of Experiments 1-4. Slopes for Condition 0/105/-150 of Experiments 2 and 3, Condition 15/120 of Experiment 3, and Experiment 4 reflect only the specified initial trials of the surprise block. 
times were constant across orientations. This suggests that subjects rotated input shapes along the shortest path that would match them with stored representations, which in the case of mirror-reversed shapes consists of a constant $180^{\circ}$ flip in depth.

These results are inconsistent with the hypothesis that complex shape recognition is accomplished by matching orientation-independent representations such as object-centered descriptions. Such a hypothesis would predict that the slope reduction that comes with practice at recognizing shapes at a specific set of orientations should transfer to new orientations, which it does not. They also falsify the conjecture that mental rotation is used only when the task requires discriminating handedness. Not only did our tasks not require handedness to be assigned, but it is unlikely that subjects surreptitiously tried to determine handedness: when the task made handedness irrelevant in principle by equating standard and reversed patterns, rotation to the nearest practice orientation still occurred. Finally, they are inconsistent with the hypothesis that the representations used in shape recognition are handedness-free: in Experiment 1, subjects profited from their experience in classifying the handedness of specific shapes in specific orientations and came to make such judgments for those shapes without mental rotation, and in Experiments 3 and 4, when recognizing standard and mirror-reversed shapes, they computed efficient paths of rotation specific to each version. These findings show that the representations used in recognition are specific to a shape in a particular orientation and a particular handedness. The representations thus are concrete or pictorial, in the sense that they are specific to the local arrangement of the objects' parts in the visual field at a particular viewing orientation. Recognition can be achieved by aligning an image of the observed object with a representation of the object at one of several stored orientations. Mismatches in orientation are compensated for by a process acting upon the concrete depiction of the observed object which requires more time for greater amounts of mismatch, presumably the continuous image transformation process called mental rotation.

\section{IMPLICATIONS FOR THE GENERAL PROBLEM OF SHAPE RECOGNITION}

As a number of authors have pointed out (e.g., Biederman, 1987; Takano, 1989; Ullman, 1986), there is probably more than one path to object recognition. Many natural objects can be identified by surface texture, local features, or the presence of some set of basic geometric solids attached in simple ways (Biederman, 1987). Our stimuli were designed to be distinguishable by their internal two-dimensional spatial configuration alone, cutting off the simpler routes to recognition, and they may be 
somewhat unusual in that regard. Although our conclusions are therefore restricted to the ability to recognize objects that differ only in the multidimensional spatial arrangement of their parts, this is a particularly interesting computational problem, and how and when people solve it is an important issue in cognitive science (Marr, 1982).

Our major conclusion is that objects seem to be represented for visual recognition by this route in multiple orientation-specific, handednessspecific representations, and that when they match an input shape at some orientation, they trigger a transformation process that aligns the observed object with one of these representations via the shortest path. This is at odds with Marr and Nishihara's (1978) widely accepted arguments that shape recognition uses viewpoint-invariant object-centered descriptions. But several empirical, physiological, ecological, and computational considerations are consistent with there being an important role for a multiple-views-plus-transformation mechanism.

The empirical literature on when human perceivers are successful at shape recognition (putting aside the question of how it is accomplished) shows that orientation-independent recognition is the exception, not the rule. In general, if a shape is tilted and perceivers do not know it, the shape looks different and they fail to recognize it (Rock, 1973, 1974, 1983). When people know or have perceptual cues telling them that a shape may be misoriented, recognition can occur, but it is far more difficult than in the case of upright objects, especially for human faces and other classes of familiar objects with subtle shape differences (Diamond \& Carey, 1986). The recognition of objects across changes of orientation in depth has not been studied in as much detail as the effects of tilts and inversions, but Rock, di Vita, and Barbeito (1981) and Rock and Di Vita (1987) have shown that people fail to recognize certain complex curved shapes when they are rotated in depth with respect to their viewpoint. Even for familiar objects, there is a preferred view or set of views at which the object is most easily recognized (Palmer et al. 1981). Thus the literature on shape recognition suggests that the storage of one or more specific views of an object at an upright orientation with respect to the viewer is the default recognition mechanism. Recognition across a full range of orientations appears to require the triggering of an additional, more difficult operation, which we have suggested is mental rotation.

It should not be completely surprising that shapes are often best recognized in a single orientation with respect to the upright. Gravity is a major force affecting objects above a certain size (Haldane, 1927/1985), and many kinds of objects tend to assume a constant orientation with respect to it. Geological objects such as mountains are formed by gravityrelated processes and are too large to be moved thereafter; many mediumto-large sized terrestrial living things evolve to be able to maintain a 
constant orientation with respect to gravity; and many human artifacts (e.g., dwellings, furniture, appliances) are designed to remain stationary in the orientation at which humans, themselves usually mono-oriented with respect to gravity, can most easily interact with them. The fact that human perceivers maintain a preferred orientation with respect to gravity means that viewer-aligned representations of mono-oriented objects will very often match those stored in memory. Of course neither objects nor perceivers are so constrained in their orientation about their vertical axes. But large stationary objects are often approached from a single direction, and in many other cases multiple views can be stored by walking around large objects or manipulating small ones. This means that one or more viewer-specific representations can successfully match input objects in many circumstances. In other circumstances, involving arbitrarily moveable objects (e.g., tools in a pile), it is not implausible that mental transformations or recognition by routes other than global geometric configuration could be used in those cases where successful recognition takes place at all.

Certain findings from neuroscience are compatible in a very general way with the hypothesis that recognition uses multiple viewpoint-specific representations that are related to the typical ecological relationship between the perceiver and objects in the world. Single-cell recording studies have demonstrated the existence of neurons in monkeys that respond to monkey faces in upright orientations and other cells that respond to monkey faces in upside-down orientations. In sheep, however, only cells that respond to upright sheep faces have been found (Kendrick \& Baldwin, 1987). The difference, the authors propose, is related to the fact that monkeys, which are arboreal, often view other monkeys upside down but sheep virtually never view other sheep upside down (Kendrick \& Baldwin, 1987). Neuronal sensitivity to orientation about the vertical axis has also been documented: Perrett, Mistlin, and Chitty (1987) have found separate cells in monkeys maximally sensitive to full-face views of faces and other cells maximally sensitive to profiles. In humans, certain kinds of brain damage lead to loss of the ability to recognize unusual views of an object, while recognition of familiar views is preserved (Warrington \& Taylor, 1978).

There are also computational arguments against the use of objectcentered descriptions in shape recognition and favoring the use of some kind of alignment algorithm. Ullman (1986) pointed out certain difficulties for the process of creating object-centered descriptions of arbitrary input shapes. For example it is often unclear how to decompose an object into a consistent set of parts, how to find a reference frame centered upon the object in a consistent way, and how to deal with objects for which different parts are obscured in different views. Ullman (1986) has outlined a 
computational model of object recognition that relies on the alignment of "pictorial descriptions" of objects to orientation-specific representations. His proposal is designed to deal with three traditional hurdles to using specific views plus transformations.

The first problem is how perceivers know, before they recognize an object, what direction to rotate it in and by how much. Ullman suggests that recognition processes could use an "alignment key": a cue to an object's orientation in the input image that is independent of its identity. A well-defined axis or a small set of landmarks, such as deep concavities or other distinctive features, are examples of alignment keys. Ullman presents a theorem that if a perceiver can find any three noncollinear landmarks on an input shape that are also defined in a stored 3D representation, the landmarks' $2 \mathrm{D}$ image coordinates are sufficient to compute the rotation, translation, and size scaling that would be needed to bring the input into alignment with the stored model. ${ }^{5}$ The second problem is how a perceiver knows which stored representations to align the input shape with (or vice versa). One possibility is to compare the alignment keys of the input object with all stored representations, execute the necessary alignments, and compare the resulting superimpositions in parallel. A more efficient alternative (Ullman, personal communication) would be to use an overconstrained alignment key. Whereas three landmarks in an image can always be aligned with three corresponding ones in a stored model via a combination of rotation, translation, and size scaling, this is not true for four or more landmarks. Thus a perceiver can try to align sets of four or more landmarks in an image with those in a stored model as closely as possible, employing something like a least-squares algorithm, and use the resulting goodness-of-fit measure as an indication of whether the alignment of the image with that particular model is worth carrying out. Something like this procedure could help resolve a paradox often noted in the mental rotation literature (Corballis et al., 1978; Parsons, 1987a, b; Shepard \& Cooper, 1982): people need to recognize an object before mentally rotating it in order know what direction and distance to rotate it, so how could they mentally rotate it in order to recognize it? The answer is that a tiny fraction of the shape information in an input objectas little as the positions of four landmarks-can be sufficient to know which stored objects to align it with and which alignment transformations are needed.

The third problem is how to measure the degree of match between input

\footnotetext{
${ }^{5}$ Why then do people sometimes fail to recognize misoriented familiar shapes when they are unaware that the shapes are rotated (Rock, 1973, 1983)? Perhaps this stems from a failure to label orientation-free alignment keys in stored shape representations or a failure to notice alignment keys in input shapes.
} 
and stored representations after the alignment is effected. Point-by-point template matching is problematic because trivial variations in an object's surface, or minor inaccuracies in the representation, can cause perceptually equivalent shapes to fail to match. However, if some of the local sections of an object are categorized (both in the input and the stored models) using coarser descriptors such as "wiggly line segment," much of this variation can be bypassed. The global arrangement of parts would be "pictorial" in the sense that metric information about their positions is preserved, but each of the parts would be represented by a descriptive label (in addition to its exact spatial contour) and part-by-part matches subsequent to the overall alignment process could be computed over them. Thus orientation-invariant descriptions may have a role in the representation of part identity, but not in the representation of the geometry of the shape as a whole.

Huttenlocher and Ullman (1987) present some operational examples of this "alignment of pictorial descriptions" model that successfully recognizes objects in arbitrary orientations in three dimensions from a single two-dimensional view. Lowe (1987) has devised similar models, in which a small number of "non-accidental" image features are used to align the image with stored models and a "viewpoint-consistency constraint" is used to assess whether the rest of the details of the input and model match. Considered in the context of these computational models and empirical evidence from psychology and neuroscience, the data we have presented support a theory of complex shape recognition giving an important role to multiple stored views plus mental transformations.

\section{REFERENCES}

Biederman, I. (1987). Recognition-by-components: A theory of human image understanding. Psychological Review, 94(2), 115-147.

Carpenter, P. A., \& Just, M. A. (1978). Eye fixations during mental rotation. In J. W. Senders, D. F. Fisher, and R. A. Monty (Eds.), Eye movements and the higher psychological functions. Hillsdale, $\mathrm{NJ}$ : Erlbaum.

Cooper, L. A. (1975). Mental rotation of random two-dimensional shapes. Cognitive Psychology, 7, 20-43.

Cooper, L. A. (1976). Demonstration of a mental analog of an external rotation. Perception \& Psychophysics, 19, 296-302.

Cooper, L. A., \& Shepard, R. N. (1973). Chronometric studies of the rotation of mental images. In W. G. Chase (Ed.), Visual information processing. New York: Academic Press.

Corballis, M. C. (1988). Recognition of disoriented shapes. Psychological Review, 95(1), 115-123.

Corballis, M. C., \& Cullen, S. (1986). Decisions about the axes of disoriented shapes. Memory \& Cognition, 14(1), 27-38.

Corballis, M. C., \& McLaren, R. (1984). Winding one's Ps and Qs: Mental rotation and mirror-image discrimination. Journal of Experimental Psychology: Human Perception and Performance, 10(2), 318-327. 
Corballis, M. C., \& Nagourney, B. A. (1978). Latency to categorize disoriented alphanumeric characters as letters or digits. Canadian Journal of Psychology, 32(3), 186-188.

Corballis, M. C., Zbrodoff, N. J., Shetzer, L. I., \& Butler, P. B. (1978). Decisions about identity and orientation of rotated letters and digits. Memory \& Cognition, 6, 98-107.

Diamond, R., \& Carey, S. (1986). Why faces are and are not special: an effect of expertise. Journal of Experimental Psychology: General, 115(2), 107-117.

Eley, M. G. (1982). Identifying rotated letter-like symbols. Memory \& Cognition, 10(1), $25-32$.

Haldane, J. B. S. (1985), On being the right size. In J. Maynard Smith (Ed.), On being the right size and other essays. Oxford: Oxford University Press. (Original work published 1927)

Hinton, G. E., \& Parsons, L. M. (1981). Frames of reference and mental imagery. In J. Long and A. Baddeley (Eds.), Attention and performance IX. Hillsdale, NJ: Erlbaum.

Huttenlocher, D. P., \& Ullman, S. (1987). Recognizing rigid objects by aligning them with an image. M.I.T. A.I. Memo, 937.

Jolicoeur, P. (1985). The time to name disoriented natural objects. Memory \& Cognition, 13(4), 289-303.

Jolicoeur, P., \& Landau, M. J. (1984). Effects of orientation on the identification of simple visual patterns. Canadian Journal of Psychology, 38(1), 80-93.

Kaushall, P., \& Parsons, L. M. (1981). Optical information and practice in the discrimination of 3-D mirror-reflected objects. Perception, 10, 545-562.

Kendrick, K. M., \& Baldwin, B. A. (1987). Cells in temporal cortex of conscious sheep can respond preferentially to the sight of faces. Science, 236, 448-450.

Koriat, A., \& Norman, J. (1985). Mental rotation and visual familiarity. Perception \& Psychophysics, 37, 429-439.

Lowe, D. G. (1987). Three-dimensional object recognition from single two-dimensional images. Artificial Intelligence, 31, 355-395.

Marr, D. (1982). Vision: A Computational Investigation into the Human Representation and Processing of Visual Information. San Francisco: Freeman.

Marr, D., \& Nishihara, H. K. (1978). Representation and recognition of the spatial organization of three-dimensional shapes. Proceedings Royal Society of London. B, 200, 269-294.

Metzler, J., \& Shepard, R. N. (1974). Transformational studies of the internal representation of three-dimensional objects. In R. L. Solso (Ed.), Theories of cognitive psychology: The Loyola symposium. Potomac, MD: LEA.

Palmer, S. E. (1975). Visual perception and world knowledge: Notes on a model of sensorycognitive interaction. In D. A. Norman and D. E. Rumelhart (Eds.), Explorations in cognition. Hillsdale, NJ: Erlbaum.

Palmer, S., Rosch, E., \& Chase, P. (1981). Canonical perspective and the perception of objects. In J. Long and A. Baddeley (Eds.), Attention and performance IX. Hillsdale, NJ: Erlbaum.

Parsons, L. M. (1987a). Imagined spatial transformation of one's body. Journal of Experimental Psychology: General, 116(2), 172-191.

Parsons, L. M. (1987b). Imagined spatial transformations of one's hands and feet. Cognitive Psychology, 19, 178-241.

Parsons, L. M. (1987c). Visual discrimination of abstract mirror-reflected three-dimensional objects at many orientations. Perception \& Psychophysics, 42(1), 49-59.

Perrett, D. I., Mistlin, A. J., \& Chitty, A. J. (1987). Visual neurones responsive to faces. Trends in Neurosciences, 10(9), 358-364.

Pinker, S. (1984). Visual cognition: An introduction. Cognition, 18, 1-63.

Robertson, L. C., Palmer, S. E., \& Gomez, L. M. (1987). Reference frames in mental 
rotation. Journal of Experimental Psychology: Learning, Memory, and Cognition, 13(3), 368-379.

Rock, I. (1973). Orientation and form. New York: Academic Press.

Rock, I. (1974). The perception of disoriented figures. Scientific American, 230(Jan.), 78-85.

Rock, I. (1983). The logic of perception. Cambridge, MA: The MIT Press.

Rock, I., \& Di Vita, J. (1987). A case of viewer-centered object perception. Cognitive Psychology, 19, 280-293.

Rock, I., Di Vita, J., \& Barbeito, R. (1981). The effect on form perception of change of orientation in the third dimension. Journal of Experimental Psychology: Human Perception and Performance, 7(4), 719-732.

Shepard, R. N. (1984). Ecological constraints on internal representation: Resonant kinematics of perceiving, imagining, thinking, and dreaming. Psychological Review, 91, 417-447.

Shepard, R. N., \& Cooper, L. A. (1982). Mental images and their transformations. Cambridge, MA: The MIT Press.

Shinar, D., \& Owen, D. H. (1973). Effects of form rotation on the speed of classification: The development of shape constancy. Perception \& Psychophysics, 14(1), 149-154.

Simion, F., Bagnara, S., Roncato, S., \& Umilta, C. (1982). Transformation processes upon the visual code. Perception \& Psychophysics, 31(1), 13-25.

Takano, Y. (1989). Perception of rotated forms: a theory of information types. Cognitive Psychology, 21, 1-59.

Tarr, M. J. (1989). Orientation-dependence in three-dimensional object recognition. Unpublished doctoral dissertation, Massachusetts Institute of Technology.

Ullman, S. (1986). An approach to object recognition: Aligning pictorial descriptions. M.I.T. A.I. Memo, 931.

Warrington, E. K., \& Taylor, A. M. (1978). Two categorical stages of object recognition. Perception, 7, 695-705.

White, M. J. (1980). Naming and categorization of tilted alphanumeric characters do not require mental rotation. Bulletin of the Psychonomic Society, 15(3), 153-156.

(Accepted November 22, 1988) 